\title{
Distributed Joint Source Coding and Trellis Coded Modulation for Symbol-Based Markov Sources
}

\author{
Mohd Azri Mohd Izhar, Member, IEEE, Abdulah Jeza Aljohani, Member, IEEE, Soon Xin Ng, Senior \\ Member, IEEE, and Lajos Hanzo, Fellow, IEEE
}

\begin{abstract}
A distributed joint source-channel coding scheme based on a unity-rate code (URC)-assisted trellis coded modulation (TCM) is proposed, which exploits the spatio-temporal correlation of symbol-based sources. More specifically, asymmetric distributed source coding of two spatially correlated Markov sources is considered, where one of the sources is assumed to be perfectly decoded and to be available at the receiver of the other source as side information. In order to exploit the temporal correlation statistics, an iterative decoding process exchanging extrinsic information between the amalgamated URC-assisted TCM and a soft-symbol source decoder employing a modified symbol-based maximum a posteriori algorithm is invoked. Furthermore, the Slepian-Wolf (SW) bound of symbol-based sources having spatiotemporal correlation is derived and the benefits of exploiting the spatio-temporal correlation using the proposed coding scheme are demonstrated by our extrinsic information transfer chart analysis. It is shown from our simulation results that upon exploiting the spatio-temporal correlation of the sources, the proposed coding scheme is capable of operating within 0.02 bit of the SW bound.
\end{abstract}

Index Terms-Joint source-channel coding, distributed source coding, distributed source-channel coding, coded modulation, iterative decoding, Slepian-Wolf coding.

\section{INTRODUCTION}

$\mathbf{M}$ ULTI-NODE communication networks such as wireless sensor networks support a number of spatially distributed nodes cooperatively working together for various purposes, e.g. environmental monitoring and recording. They exhibit promising features, but their main challenge is the limited power available for the nodes to operate. Thus, saving energy is crucial. Distributed source coding (DSC) constitutes a promising solution, since it is capable of exploiting the correlation of the sources for reducing the overall energy

Copyright (c) 2017 IEEE. Personal use of this material is permitted. However, permission to use this material for any other purposes must be obtained from the IEEE by sending a request to pubs-permissions@ieee.org.

M. A. M. Izhar is with the Wireless Communication Center and Ubiquitous Broadband Access Network (U-BAN) Group, UTM Razak School of Engineering and Advanced Technology, Universiti Teknologi Malaysia, Jalan Sultan Yahya Petra, Kuala Lumpur, 54100, Malaysia. (e-mail: mohdazri.kl@utm.my).

A. J. Aljohani is with the Umm Alqura University, Mecca, Saudi Arabia. (e-mail: ajjoahni@uqu.edu.sa).

S. X. Ng and L. Hanzo are with the School of Electronics and Computer Science, University of Southampton, Southampton, SO17 1BJ, United Kingdom. (email: $\{$ sxn, lh $\} @$ ecs.soton.ac.uk).

This work was supported in part by the Malaysian Ministry of Higher Education, in part by the Universiti Teknologi Malaysia, in part by the European Research Council through the Advanced Fellow Grant, in part by the Roya Society's Wolfson Research Merit Award, and in part by the Engineering and Physical Sciences Research Council under Grant EP/L018659/1. The research data for this paper is available at http://doi.org/10.5258/SOTON/D0374. consumption [1]-[27]. Furthermore, it shifts the power-hungry signal processing to the central base station, whilst ensuring a low complexity at the sensor nodes.

The theoretical framework of DSC was conceived in the seminal treatise of Slepian and Wolf [1] for lossless sources and later it was followed by Wyner and Ziv [2] for the case of lossy sources. In the late 1990s, a pioneering practical DSC solution based on the syndrome of channel codes was introduced by Pradhan and Ramchandran [3], [4]. In order to achieve a performance close to the Slepian-Wolf (SW) theoretical limit, DSC schemes based on capacity-approaching codes, e.g., turbo codes [9]-[11] and low-density-parity-check (LDPC) codes [12]-[14] were developed. Afterwards, a considerable amount of work has been dedicated to addressing the problem of transmitting a pair of correlated sources over noisy channels that led to the development of distributed joint source-channel coding (DJSCC) schemes aiming to perform close to the theoretical limit established by the combination of the Shannon and SW theorems [15]-[23]. A DSJCC scheme relying on a joint turbo equalizer and decoder scheme was developed for the so-called asymmetric ${ }^{1}$ scenario in [17] and for the symmetric scenario in [19]. Recently, a bandwidth-efficient DJSCC scheme based on turbo trellis coded modulation (TTCM) was proposed in [22], which exhibits a significantly lower decoding complexity than those in [17], [19]. However, similar to all of the abovementioned investigations, the correlation of binary rather than non-binary sources was considered. However, the symbol-tobit conversion of non-binary sources may reduce the inherent source-symbol redundancy and subsequently may result in a reduced performance improvement. In this paper, we extend the scenario of binary sources [22] to a more general symbolbased DJSCC scheme capable of handling both binary and non-binary sources.

Apart from their spatial correlation, the information gathered by the sensor nodes may also be correlated in the time domain due to the nature of the physical phenomena that changes with time. The correlation of temporally-correlated sources can be exploited in conjunction with channel decoding using soft-bit source decoding [28]-[34] and by modifying the channel decoding algorithm to incorporate the source correlation knowledge [35]-[39]. The temporal correlation statistics can also be jointly exploited along with the spatial inter-source correlation for further enhancing the overall performance of

\footnotetext{
${ }^{1}$ Asymmetric DSC considers different compression rates between the spatially correlated sources and an extreme case of asymmetric DSC is the decoding with perfect side information. Meanwhile, symmetric DSC considers the same compression rate between the spatially correlated sources.
} 
the network. However, as compared to the number of studies exploiting either the spatial or temporal correlation, there is a paucity of contributions on handling the spatio-temporal correlation [24]-[27]. These investigations have demonstrated that further performance enhancements can be achieved upon jointly exploiting both the spatial and temporal correlations compared to exploiting only the spatial correlation. However, the schemes proposed in [24]-[27] were designed for binary sources and for binary phase-shift keying (BPSK) transmission over either additive white Gaussian noise (AWGN) channels [24]-[26] or over Rayleigh fading channels [27].

Against this background, we introduce a bandwidth-efficient symbol-based DJSCC scheme for exploiting the spatiotemporal correlation which is specifically designed for the asymmetrical DSC scenario, where the spatially correlated information gleaned from the second source is assumed to be perfectly decoded and readily available at the receiver of the first source as side information. An $M$-ary symmetric channel ( $M$-SC) is used for modeling the spatial correlation and the sources are characterized by a first-order Markov process in terms of the temporal correlation. Instead of using a TTCMbased DSC scheme as in [22], we employ a serial concatenated unity-rate code (URC) and a TCM scheme. This is justified, because we will show later in the paper that the proposed scheme has a higher performance advantage than the TTCMbased scheme, despite imposing a lower decoding complexity. Hence, our novel contributions can be summarized as follows:

- We propose a distributed joint source coding and URCassisted TCM scheme exploiting the spatio-temporal source correlation (DJSUTCM-ST) that is capable of achieving a near-SW/Shannon (SW/S) performance. An iterative decoding process is invoked for exchanging the extrinsic information between the URC-assisted TCM and a source decoder, where the source symbol correlation obeys a first-order Markov model. Extrinsic information transfer (EXIT) chart analysis is invoked for finding the TCM coding parameters for various sources having both different number of bits/symbol and spatiotemporal correlation. The proposed scheme is capable of operating at a high code rate in conjunction with high-order modulation. Hence, it is well-suited for highrate communication applications such as video streaming. We further develop the BPSK-aided AWGN scenario of [24]-[26] to more realistic uncorrelated Rayleigh fading channels and high-order modulation schemes.

- Additionally, in contrast to the DJSCC schemes of [24][27] that are only capable of exploiting the spatiotemporal correlation of binary sources, we consider a generalized symbol-based DJSCC for exploiting the spatio-temporal correlation of symbol-based sources, which can be binary or non-binary. A symbol-based maximum a-posteriori (MAP) algorithm [40] is invoked for URC and TCM decoding in order to avoid information loss due to bit-to-symbol or symbol-to-bit conversion between the source and channel decoders.

- A new SW bound is derived for sources exhibiting spatio-temporal correlation based on the M-SC model

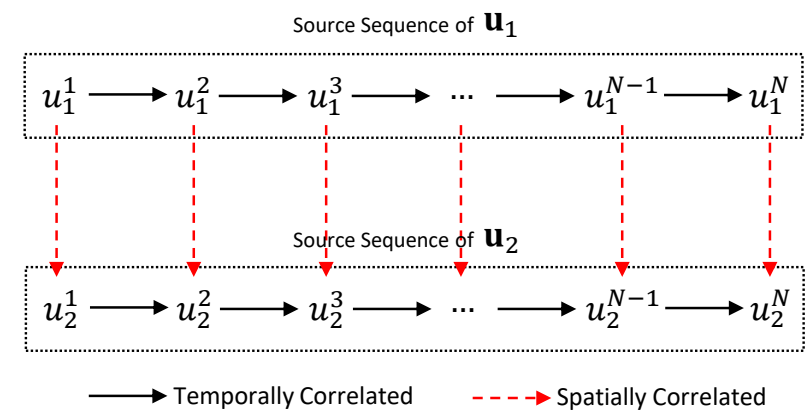

Fig. 1. Spatio-temporal correlation of a pair of source sequences.

and the first-order Markov chain, which are used for characterizing the spatial correlation and the temporal correlation of the sources, respectively. Based on this new SW bound, the SW/S limit is determined by using the discrete-input continuous-output memoryless channel (DCMC) capacity [41].

- We invoke the low complexity MAP decoding algorithm of [33] for the decoding of first-order Markov sources. Since the information exchanged by our proposed iterative decoding scheme is in the form of symbol probabilities, the MAP algorithm [33] originally developed for softbit source decoding is modified to take into account the symbol probabilities both at its input and output.

The proposed coding scheme might find fruitful applications in high-rate multimedia transmission over multi-node vehicular communication networks. Amongst the potential communication applications in a vehicular context are multispectral and hyperspectral imaging for detecting images acquired by multiple satellites or aircraft [42], [43], for environmental and traffic monitoring by vehicular sensor networks [44], [45], for various multimedia-rich applications in vehicular adhoc networks [46]-[49], and for real-time security monitoring around a video surveillance vehicle using a pair of cameras [50].

The remainder of this paper is organized as follows. The spatially and temporally correlated source models are described in Section II. The proposed joint decoding technique relying on the symbol-based source decoder conceived for the decoding of first-order Markov sources is detailed in Section III. Our simulation results characterizing the proposed technique are presented in Section IV, while our concluding remarks are provided in Section V.

\section{Spatio-Temporal Source Correlation Model}

A pair of $R_{\mathrm{s}}$ bits/symbol source sequences having a length of $N$ symbols, namely $\mathbf{u}_{1}=\left\{u_{1}^{1}, u_{1}^{2}, \ldots, u_{1}^{N}\right\}$ and $\mathbf{u}_{2}=$ $\left\{u_{2}^{1}, u_{2}^{2}, \ldots, u_{2}^{N}\right\}$ are considered, as depicted in Fig. 1. Each symbol assumes one of the $M=2^{R_{\mathrm{s}}}$ legitimate values from the set of $\{0,1,2, \ldots, M-1\}$. Both sequences are spatially correlated and an $M$-SC is used for modeling the spatial correlation. In contrast to the binary symmetric channel (BSC) having $(2 \times 2)$ possible state transitions, in $M$-SC there are 
$(M \times M)$ state transitions and the corresponding transition probabilities can be represented by an $(M \times M)$-element matrix as

$$
\mathbf{A}^{\mathrm{sp}}=\left[a_{i, j}^{\mathrm{sp}}\right]_{M \times M} \quad i, j \in\{0,1, \ldots, M-1\} .
$$

The component $a_{i, j}^{\mathrm{sp}}$ of the matrix is defined as the transition probability from source $u_{1}^{t}$ to $u_{2}^{t}$, i.e. $P\left(u_{2}^{t}=j \mid u_{1}^{t}=i\right.$ ) (or correspondingly from $u_{2}^{t}$ to $u_{1}^{t}$, i.e. $P\left(u_{1}^{t}=j \mid u_{2}^{t}=i\right)$ ) at a time index $t$ for $t=\{1,2, \ldots, N\}$ and it is is given by

$$
a_{i, j}^{\mathrm{sp}}= \begin{cases}p_{\mathrm{sp}}=1-p_{\mathrm{e}} & \text { for } j=i \\ q_{\mathrm{sp}}=\frac{p_{\mathrm{e}}}{M-1} & \text { for } j \neq i,\end{cases}
$$

where $p_{\mathrm{e}}$ is the error probability encountered in the spatial domain. The spatial correlation depends on the $p_{\mathrm{e}}$ value, which determines the values of $p_{\mathrm{sp}}$ and $q_{\mathrm{sp}}$ in (2). The correlation is zero when we have $p_{\mathrm{sp}}=q_{\mathrm{sp}}$ and as the difference between $p_{\mathrm{sp}}$ and $q_{\mathrm{sp}}$ increases, the correlation becomes higher. The spatial correlation coefficient $\rho_{\mathrm{sp}}$ can be determined from the $p_{\mathrm{sp}}$ value using

$$
\rho_{\mathrm{sp}}=\frac{p_{\mathrm{sp}}-1 / M}{1-1 / M} .
$$

Again, first-order Markov sources model the temporal correlation both at Source 1 and Source 2. For Source 1, the value of $u_{1}^{t}$ at a time index $t$ depends on the previous value $u_{1}^{t-1}$ and this transition can be represented by an $(M \times M)$ transition probability matrix

$$
\mathbf{A}^{\text {te }}=\left[a_{i^{\prime}, i}^{\mathrm{te}}\right]_{M \times M} \quad i^{\prime}, i \in\{0,1, \ldots, M-1\},
$$

where $a_{i^{\prime}, i}^{\text {te }}=P\left(u_{1}^{t}=i \mid u_{1}^{t-1}=i^{\prime}\right)$. In this work, we assume that for $i=i^{\prime}$, the same transition probability value is specified for $i \in\{0,1, \ldots, M-1\}$ and this transition probability is defined as $p_{\mathrm{te}}$. On the other hand, for $i \neq i^{\prime}$, it is assumed that we have $a_{i^{\prime}, i}^{\text {te }}=q_{\mathrm{te}}=\left(1-p_{\mathrm{te}}\right) /(M-1)$. The temporal correlation coefficient of Source 1, $\rho_{\text {te }}$ can be calculated as

$$
\rho_{\mathrm{te}}=\frac{p_{\mathrm{te}}-1 / M}{1-1 / M},
$$

and likewise for Source 2, the temporal correlation is denoted as $\rho_{\text {te2 } 2}$, which can be determined when replacing $p_{\text {te }}$ in (5) by $p_{\mathrm{te} 2}$, where $p_{\mathrm{te} 2}$ is the identical transition probability corresponding to $P\left(u_{2}^{t}=i \mid u_{2}^{t-1}=i\right)$ for $i \in\{0,1, \ldots, M-1\}$. Similar to Source 1 , for $i \neq i^{\prime}$, it is assumed that $P\left(u_{2}^{t}=\right.$ $\left.i \mid u_{2}^{t-1}=i^{\prime}\right)=q_{\mathrm{te} 2}=\left(1-p_{\mathrm{te} 2}\right) /(M-1)$. Given the set of transition probabilities corresponding to the spatial correlation and to the temporal correlation of Source 1, the transition probability associated with the temporal correlation of Source 2 can be formulated as

$P\left(u_{2}^{t} \mid u_{2}^{t-1}\right)=\sum_{u_{1}^{t}, u_{1}^{t-1}} P\left(u_{2}^{t} \mid u_{1}^{t}\right) \cdot P\left(u_{1}^{t} \mid u_{1}^{t-1}\right) \cdot P\left(u_{1}^{t-1} \mid u_{2}^{t-1}\right)$,

and from (6), we have $p_{\mathrm{te} 2}=p_{\mathrm{te}} \cdot p_{\mathrm{sp}}{ }^{2}+2 \cdot(M-1) \cdot p_{\mathrm{sp}} \cdot q_{\mathrm{te}}$. $q_{\mathrm{sp}}+(M-1) \cdot p_{\mathrm{te}} \cdot q_{\mathrm{sp}}{ }^{2}+\left(M^{2}+2-3 \cdot M\right) \cdot q_{\mathrm{te}} \cdot{q_{\mathrm{sp}}}^{2}$.

In general, the entropy rate of a first-order Markov source with previous state $i^{\prime}$ and current state $i$ can be calculated by $H=-\sum_{i^{\prime}, i \in\{0,1, \ldots, M-1\}} \mu_{i^{\prime}} a_{i^{\prime}, i} \log _{2} a_{i^{\prime}, i}$, where $a_{i^{\prime}, i}$ is the transition probability and $\mu_{i^{\prime}}$ represents the stationary

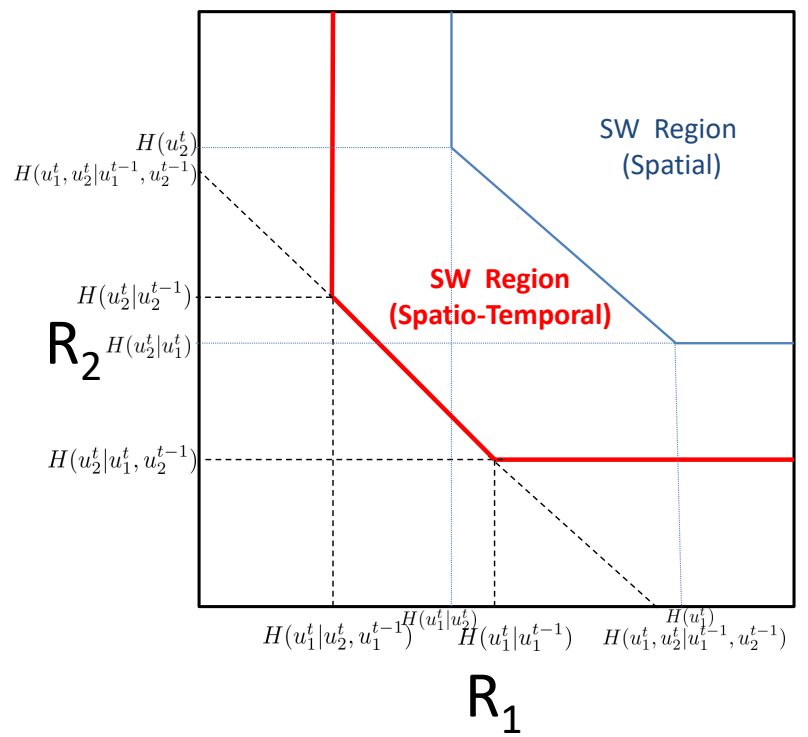

Fig. 2. Achievable rate region for sources exhibiting spatio-temporal correlation assuming $\mathcal{H}\left(\mathbf{u}_{1}\right)>\mathcal{H}\left(\mathbf{u}_{1} \mid \mathbf{u}_{2}\right)$ and $\mathcal{H}\left(\mathbf{u}_{2}\right)>\mathcal{H}\left(\mathbf{u}_{2} \mid \mathbf{u}_{1}\right)$.

state distribution [51]. Based on our model and on the assumptions considered above, the stationary state distribution for the Markov sources considered is given by $1 / M$ for $i^{\prime} \in\{0,1, \ldots, M-1\}$. Therefore, the conditional entropy rate of $\mathbf{u}_{1}$ given $\mathbf{u}_{2}$, as well as the entropy rate of $\mathbf{u}_{1}$ and the entropy rate of $\mathbf{u}_{2}$ can be determined from

$$
\begin{aligned}
\mathcal{H}\left(\mathbf{u}_{1} \mid \mathbf{u}_{2}\right) & =H\left(u_{1}^{t} \mid u_{2}^{t}\right), \\
& =-p_{\mathrm{sp}} \cdot \log _{2}\left(p_{\mathrm{sp}}\right)-(M-1) \cdot q_{\mathrm{sp}} \cdot \log _{2}\left(q_{\mathrm{sp}}\right), \\
\mathcal{H}\left(\mathbf{u}_{1}\right)= & H\left(u_{1}^{t} \mid u_{1}^{t-1}\right), \\
= & -p_{\mathrm{te}} \cdot \log _{2}\left(p_{\mathrm{te}}\right)-(M-1) \cdot q_{\mathrm{te}} \cdot \log _{2}\left(q_{\mathrm{te}}\right),
\end{aligned}
$$

and

$$
\begin{aligned}
\mathcal{H}\left(\mathbf{u}_{2}\right) & =H\left(u_{2}^{t} \mid u_{2}^{t-1}\right), \\
& =-p_{\mathrm{te} 2} \cdot \log _{2}\left(p_{\mathrm{te} 2}\right)-(M-1) \cdot q_{\mathrm{te} 2} \cdot \log _{2}\left(q_{\mathrm{te} 2}\right),
\end{aligned}
$$

respectively, where $H\left(u_{1}^{t} \mid u_{2}^{t}\right)$ is the conditional entropy of $u_{1}^{t}$ given $u_{2}^{t}$, which $H\left(u_{1}^{t} \mid u_{1}^{t-1}\right)$ is the entropy of $u_{1}^{t}$ given $u_{1}^{t-1}$ and $H\left(u_{2}^{t} \mid u_{2}^{t-1}\right)$ is the entropy of $u_{2}^{t}$ given $u_{2}^{t-1}$. It is worth noting that due to the symmetrical nature of the $M$-SC model, we have $\mathcal{H}\left(\mathbf{u}_{2} \mid \mathbf{u}_{1}\right)=\mathcal{H}\left(\mathbf{u}_{1} \mid \mathbf{u}_{2}\right)$.

\section{A. Slepian-Wolf rate region}

Considering the spatial correlation only, the SW coding theorem [1] specifies the achievable compression rates, $R_{1}$ and $R_{2}$ for Source 1 and Source 2, respectively as: $R_{1} \geq$ $\mathcal{H}\left(\mathbf{u}_{1} \mid \mathbf{u}_{2}\right), R_{2} \geq \mathcal{H}\left(\mathbf{u}_{2} \mid \mathbf{u}_{1}\right)$ and $R_{1}+R_{2} \geq \mathcal{H}\left(\mathbf{u}_{1}, \mathbf{u}_{2}\right)$. As for the spatio-temporal correlation, the achievable SW rate region is illustrated in Fig. 2, where the rate region is bounded by the following three inequalities: 


$$
\begin{aligned}
R_{1} & \geq H\left(u_{1}^{t} \mid u_{2}^{t}, u_{1}^{t-1}\right), \\
R_{2} & \geq H\left(u_{2}^{t} \mid u_{1}^{t}, u_{2}^{t-1}\right), \\
R_{1}+R_{2} & \geq H\left(u_{1}^{t}, u_{2}^{t} \mid u_{1}^{t-1}, u_{2}^{t-1}\right) .
\end{aligned}
$$

Using Bayes' rule for the conditional entropy, $R_{1}$ can be expressed as

$$
R_{1} \geq H\left(u_{2}^{t}, u_{1}^{t-1} \mid u_{1}^{t}\right)+H\left(u_{1}^{t}\right)-H\left(u_{2}^{t}, u_{1}^{t-1}\right),
$$

and by assuming that $u_{2}^{t}$ and $u_{1}^{t-1}$ are independent of each other given $u_{1}^{t}$, we have

$$
R_{1} \geq H\left(u_{2}^{t} \mid u_{1}^{t}\right)+H\left(u_{1}^{t-1} \mid u_{1}^{t}\right)+H\left(u_{1}^{t}\right)-H\left(u_{2}^{t}, u_{1}^{t-1}\right)
$$

and substitute the joint entropy $H\left(u_{2}^{t}, u_{1}^{t-1}\right)$ with $H\left(u_{2}^{t} \mid u_{1}^{t-1}\right)-H\left(u_{1}^{t-1}\right)$ to yield

$$
\begin{aligned}
R_{1} \geq & H\left(u_{2}^{t} \mid u_{1}^{t}\right)+H\left(u_{1}^{t-1} \mid u_{1}^{t}\right)+H\left(u_{1}^{t}\right)-H\left(u_{2}^{t} \mid u_{1}^{t-1}\right) \\
& -H\left(u_{1}^{t-1}\right), \\
\geq & H\left(u_{2}^{t} \mid u_{1}^{t}\right)+H\left(u_{1}^{t-1} \mid u_{1}^{t}\right)-H\left(u_{2}^{t} \mid u_{1}^{t-1}\right) .
\end{aligned}
$$

Using Bayes' rule of conditional entropy, $H\left(u_{2}^{t} \mid u_{1}^{t}\right)=$ $H\left(u_{1}^{t} \mid u_{2}^{t}\right)-H\left(u_{1}^{t}\right)+H\left(u_{2}^{t}\right)$ and $H\left(u_{1}^{t-1} \mid u_{1}^{t}\right)=H\left(u_{1}^{t} \mid u_{1}^{t-1}\right)-$ $H\left(u_{1}^{t}\right)+H\left(u_{1}^{t-1}\right),(15)$ can be rewritten as:

$$
\begin{aligned}
R_{1} \geq & H\left(u_{1}^{t} \mid u_{2}^{t}\right)+H\left(u_{1}^{t} \mid u_{1}^{t-1}\right)+H\left(u_{2}^{t}\right)+H\left(u_{1}^{t-1}\right) \\
& -2 H\left(u_{1}^{t}\right)-H\left(u_{2}^{t} \mid u_{1}^{t-1}\right), \\
\geq & \mathcal{H}\left(\mathbf{u}_{1} \mid \mathbf{u}_{2}\right)+\mathcal{H}\left(\mathbf{u}_{1}\right)+H\left(u_{2}^{t}\right)+H\left(u_{1}^{t-1}\right) \\
& -2 H\left(u_{1}^{t}\right)-H\left(u_{2}^{t} \mid u_{1}^{t-1}\right) .
\end{aligned}
$$

Furthermore, due to the symmetric and stationary nature of the Markov sources considered at both sources, $H\left(u_{2}^{t}\right)=$ $H\left(u_{1}^{t-1}\right)=H\left(u_{1}^{t}\right)=R_{\mathrm{s}}$, we have

$$
R_{1} \geq \mathcal{H}\left(\mathbf{u}_{1} \mid \mathbf{u}_{2}\right)+\mathcal{H}\left(\mathbf{u}_{1}\right)-H\left(u_{2}^{t} \mid u_{1}^{t-1}\right) .
$$

It can be noted from (10) and (17) that the term $H\left(u_{1}^{t} \mid u_{2}^{t}, u_{1}^{t-1}\right)$ can be expressed in terms of $\mathcal{H}\left(\mathbf{u}_{2} \mid \mathbf{u}_{1}\right)$ and $\mathcal{H}\left(\mathbf{u}_{1}\right)$. Similarly for $R_{2}$, the associated limit can be determined as

$$
R_{2} \geq \mathcal{H}\left(\mathbf{u}_{2} \mid \mathbf{u}_{1}\right)+\mathcal{H}\left(\mathbf{u}_{2}\right)-H\left(u_{1}^{t} \mid u_{2}^{t-1}\right) .
$$

Meanwhile, the bound for $\left(R_{1}+R_{2}\right)$ can be expressed as

$$
\begin{aligned}
R_{1}+R_{2} & \geq H\left(u_{1}^{t}, u_{2}^{t} \mid u_{1}^{t-1}, u_{2}^{t-1}\right), \\
& \geq H\left(u_{1}^{t} \mid u_{2}^{t}, u_{1}^{t-1}, u_{2}^{t-1}\right)+H\left(u_{2}^{t} \mid u_{1}^{t-1}, u_{2}^{t-1}\right),
\end{aligned}
$$

and since a first-order Markov process is considered, (19) can be simplified to

$$
R_{1}+R_{2} \geq H\left(u_{1}^{t} \mid u_{2}^{t}, u_{1}^{t-1}\right)+H\left(u_{2}^{t} \mid u_{2}^{t-1}\right) .
$$

\footnotetext{
${ }^{2}$ Sources exhibiting spatio-temporal correlation result in higher compression rates than sources exhibiting spatial-only correlation. For example, the value of $u_{1}^{t}$ depends not only on $u_{2}^{t}$ but also on $u_{1}^{t-1}$. Hence, the compression rate for $R_{1}$ is bounded by the entropy of $H\left(u_{1}^{t} \mid u_{2}^{t}, u_{1}^{t-1}\right)$. Similarly, for $R_{2}$ where the value of $u_{2}^{t}$ depends not only on $u_{1}^{t}$ but also on $u_{2}^{t-1}$. Hence, the compression rate for $R_{2}$ is bounded by the entropy of $H\left(u_{2}^{t} \mid u_{1}^{t}, u_{2}^{t-1}\right)$. For $R_{1}+R_{2}$, the value of both $u_{1}^{t}$ and $u_{2}^{t}$ depends on $u_{1}^{t-1}$ and $u_{2}^{t-1}$ due to the spatio-temporal correlation.
}

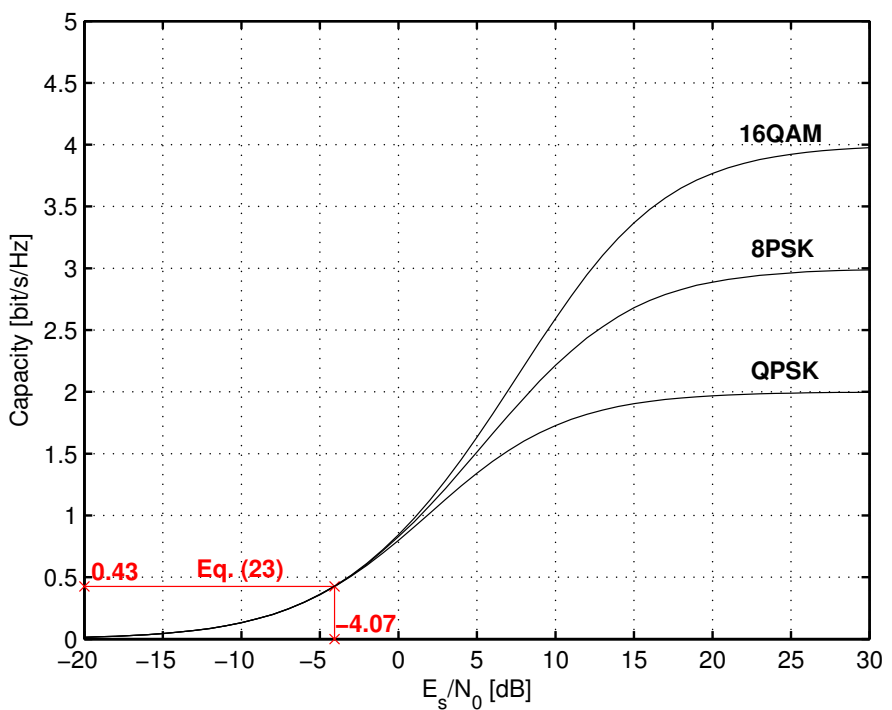

Fig. 3. QPSK, 8PSK and 16QAM DCMC capacity curves computed based on [41].

The first term of (20) is the same expression of $R_{1}$ as that given in (10). Hence, upon substituting the $R_{1}$ of (17) into (20), we arrive at

$$
\begin{aligned}
R_{1}+R_{2} & \geq \mathcal{H}\left(\mathbf{u}_{1} \mid \mathbf{u}_{2}\right)+\mathcal{H}\left(\mathbf{u}_{1}\right)-H\left(u_{2}^{t} \mid u_{1}^{t-1}\right)+H\left(u_{2}^{t} \mid u_{2}^{t-1}\right), \\
& \geq \mathcal{H}\left(\mathbf{u}_{1} \mid \mathbf{u}_{2}\right)+\mathcal{H}\left(\mathbf{u}_{1}\right)-H\left(u_{2}^{t} \mid u_{1}^{t-1}\right)+\mathcal{H}\left(\mathbf{u}_{2}\right) .
\end{aligned}
$$

\section{B. Slepian-Wolf/Shannon limit}

The information of Source 1 is transmitted over an uncorrelated Rayleigh fading channel having an ergodic channel capacity of $C=\mathbf{E}\left\{\log _{2}(1+\mathrm{SNR})\right\}$, where SNR $=|h|^{2} E_{\mathrm{s}} / N_{0}$ is the signal-to-noise ratio at the receiver input, $E_{\mathrm{s}}$ is the energy per modulated symbol, $N_{0}$ is the noise power spectral density and the expectation is taken over the random variable of the channel coefficient $h$. Based on the SW bound given of (10), the transmission reliability of $\mathbf{u}_{1}$ over a noisy channel is subject to a certain condition given by

$$
\begin{aligned}
\frac{C \cdot R_{\mathrm{s}}}{R_{\mathrm{c}} \cdot R_{\mathrm{m}}} & \geq H\left(u_{1}^{t} \mid u_{2}^{t}, u_{1}^{t-1}\right), \\
C & \geq \frac{R_{\mathrm{c}} \cdot R_{\mathrm{m}} \cdot\left(\mathcal{H}\left(\mathbf{u}_{1} \mid \mathbf{u}_{2}\right)+\mathcal{H}\left(\mathbf{u}_{1}\right)-H\left(u_{2}^{t} \mid u_{1}^{t-1}\right)\right)}{R_{\mathrm{s}}}, \\
C & \geq \eta,
\end{aligned}
$$

where $\eta$ is the overall throughput of the system, $R_{\mathrm{c}}$ is the channel coding rate and $R_{\mathrm{m}}$ is the number of bits represented by each modulation symbol. Following the terminologies used in [16], we will use the energy per generated source bit $E_{\text {so }}$ in the simulation results and the relationship of $E_{\text {so }}$ with the energy per information bit $E_{\mathrm{b}}$ as well as that of the energy per modulated symbol $E_{\mathrm{s}}$ is given by $E_{\mathrm{so}}=$ $H\left(u_{1}^{t} \mid u_{2}^{t}, u_{1}^{t-1}\right) \cdot E_{\mathrm{b}} / R_{\mathrm{s}}=E_{\mathrm{s}} /\left(R_{\mathrm{c}} R_{\mathrm{m}}\right)$. The theoretical limit, which is expressed as $\left(E_{\mathrm{so}} / N_{0}\right)_{\mathrm{lim}}$, is determined by substituting the capacity equation of $C=\mathbf{E}\left\{\log _{2}\left(1+|h|^{2} E_{\mathrm{s}} / N_{0}\right)\right\}$ into (22). The minimum $E_{\mathrm{so}} / N_{0}$ required for approaching the SW/S limit can be determined using (22) and the DCMC 
capacity curves plotted in Fig. 3 for quadrature phase shift keying (QPSK), 8-ary phase shift keying (8PSK), and 16-ary quadrature amplitude modulation (16QAM) [41].

For instance, $R_{\mathrm{s}}=2$ bits/symbol sources associated with $\rho_{\mathrm{te}}=\rho_{\mathrm{sp}}=0.8$ (corresponding to $p_{\mathrm{te}}=p_{\mathrm{sp}}=0.85$ ) having $\mathcal{H}\left(\mathbf{u}_{1} \mid \mathbf{u}_{2}\right)=\mathcal{H}\left(\mathbf{u}_{1}\right)=0.85$ bit and $H\left(u_{2}^{t} \mid u_{1}^{t-1}\right)=1.27$ bits will give $\eta=0.43$. By referring to the $8 \mathrm{PSK}$ plot of Fig. 3, this corresponding to $\left(E_{\mathrm{s}} / N_{0}\right)_{\lim }=-4.07 \mathrm{~dB}$. Then, the $\left(E_{\mathrm{so}} / N_{0}\right)_{\text {lim }}$ can be determined by $\left(E_{\mathrm{s}} / N_{0}\right)_{\text {lim }}-$ $10 \log \left(R_{\mathrm{c}} R_{\mathrm{m}}\right)=-7.08 \mathrm{~dB}$.

\section{SySTEM DESIGN}

Fig. 4 depicts the system model of the proposed DJSUTCMST scheme. ${ }^{3}$ Again, an asymmetrical communication system is considered, where the information sequence $\mathbf{u}_{1}$ of Source 1 is transmitted over a noisy channel and the spatially correlated information sequence $\mathbf{u}_{2}$ of Source 2 is assumed to be perfectly known at the decoder as side information. At Source 1, the $N$-symbol sequence $\mathbf{u}_{1}$ is first interleaved by a symbol interleaver $\Pi_{\mathrm{ST}}$ before being encoded by a TCM encoder, $\mathrm{ENC}_{\mathrm{T}}$ having a code rate of $R_{\mathrm{c}}=R_{\mathrm{s}} /\left(R_{\mathrm{s}}+1\right)$. The encoded symbol sequence $\mathbf{v}=\left\{v_{1}, v_{2}, \ldots, v_{N}\right\}$ is then interleaved by a symbol interleaver $\Pi_{\mathrm{TU}}$, encoded by a symbol-based URC encoder, $\mathrm{ENC}_{\mathrm{U}}$ [52] and modulated by an $\mathcal{M}$-ary PSK or $\mathcal{M}$ ary QAM signalling to form $\mathbf{x}=\left\{x_{1}, x_{2}, \ldots, x_{N}\right\}$, where we have $\mathcal{M}=(2 \times M)$ corresponding to a modulation scheme having $R_{\mathrm{m}}=R_{\mathrm{s}}+1$ bits/symbol. The modulated symbol sequence $\mathbf{x}$ is transmitted over an uncorrelated Rayleigh fading channel to yield the received sequence $\mathbf{y}=\left\{y_{1}, y_{2}, \ldots, y_{N}\right\}$ at the receiver. The received signal $y_{n}$ of the $n$-th symbol index of the sequence is given by

$$
y_{n}=h_{n} x_{n}+w_{n},
$$

where $h_{n}$ is a complex-valued channel coefficient and $w_{n}$ is a complex AWGN having a zero mean and a variance of $N_{0} / 2$ per dimension of the $n$-th symbol index of the sequence. ${ }^{4}$

\section{A. Proposed joint decoding technique exploiting the spatio- temporal source correlation}

Iterative decoding is invoked for exploiting the spatiotemporal correlation, as shown in Fig. 4. More explicitly, the spatial correlation is exploited using the side information gleaned from $\mathbf{u}_{2}$ is fed into the source decoder, $\mathrm{DEC}_{\mathrm{S}}$ as the a priori symbol probability $A_{\mathrm{SI}}\left(\mathbf{u}_{1}\right)$. Furthermore, in order to exploit the temporal correlation, the first-order Markov

\footnotetext{
${ }^{3}$ It is also possible to use different number of bits/symbol for the source symbol and the TCM input symbol by adding a symbol-to-symbol converter between the source and the TCM encoder, and correspondingly between the source decoder and the TCM decoder. However, this may lead to loss of precious correlation information due to the conversion and may hence degrade the performance attained. Based on our experiments, for the same correlation and a specific number of modulation level, we found that using the same symbol-length for both the source and for the TCM input symbol would achieve a better performance than that using different symbol-length. Therefore, we opt for investigating the same symbol-length $R_{\mathrm{s}}$ for the source and TCM input symbols.

${ }^{4}$ Since we consider an uncorrelated Rayleigh fading channel (also known as the fast Rayleigh fading channel), the successive channel coefficients are uncorrelated and the fading obeys a Rayleigh distribution. It is assumed that the channel coefficients are known at the receiver.
}

decoding is carried out at source decoder $\mathrm{DEC}_{\mathrm{S}}$, as detailed in Section III(B).

DEC $_{\mathrm{U}}$ of Fig. 4 receives inputs both from the channel's symbol probability $Z(\mathbf{c})$ as well as from the a priori symbol probability $A_{\mathrm{U}}\left(\mathbf{v}^{\prime}\right)$ and performs symbol-based MAP decoding for generating the extrinsic symbol probability output $E_{\mathrm{U}}\left(\mathbf{v}^{\prime}\right)$. This extrinsic output is then de-interleaved by $\Pi_{\mathrm{TU}}^{-1}$ and fed to the TCM decoder, $\mathrm{DEC}_{\mathrm{T}}$ as the a priori symbol probability $A_{\mathrm{T}}(\mathbf{v})$. Together with the a priori information $A_{\mathrm{T}}\left(\mathbf{u}_{1}^{\prime}\right)$ inferred from the source decoder $\mathrm{DEC}_{\mathrm{S}}$, symbol-based MAP decoding is performed at $\mathrm{DEC}_{\mathrm{T}}$ for producing a pair of extrinsic outputs, $E_{\mathrm{T}}(\mathbf{v})$ and $E_{\mathrm{T}}\left(\mathbf{u}_{1}^{\prime}\right)$ to be fed to $\mathrm{DEC}_{\mathrm{U}}$ and $\mathrm{DEC}_{\mathrm{S}}$, respectively. The information exchanging between $\mathrm{DEC}_{\mathrm{U}}$ and $\mathrm{DEC}_{\mathrm{T}}$ is defined as the inner iteration and it is performed until $\mathcal{I}_{\text {in }}$ number of times. Then, the extrinsic information $E_{\mathrm{T}}\left(\mathbf{u}_{1}^{\prime}\right)$ is de-interleaved by $\Pi_{\mathrm{ST}}^{-1}$ and passed to $\mathrm{DEC}_{\mathrm{S}}$ of Fig. 4 . The three inputs are processed by $\mathrm{DEC}_{\mathrm{S}}$, which are the a priori symbol probability $A_{\mathrm{S}}\left(\mathbf{u}_{1}\right)$ received from $\mathrm{DEC}_{\mathrm{T}}$, the side information $A_{\text {SI }}\left(\mathbf{u}_{1}\right)$ gleaned from Source 2 and the transition probability $p_{\text {te }}$, which is assumed to be perfectly known at the receiver.

The side information inferred from Source 2 is generated by first converting $\mathbf{u}_{2}$ to the symbol probability $A_{2}\left(\mathbf{u}_{2}\right)$, where $A_{2}\left(u_{2}^{t}=i\right)=0$ if $i \neq u_{2}^{t}$ and $A_{2}\left(u_{2}^{t}=i\right)=1$ if $i=u_{2}^{t}$ for $i=\{0,1, . ., M-1\}$. Before the a priori information $A_{2}\left(\mathbf{u}_{2}\right)$ is passed to the decoder of Source 1, it has to be corrected with the aid of the function $f_{\mathrm{c}}$ of Fig. 4, which updates the information $A_{2}\left(\mathbf{u}_{2}\right)$ as follows:

$A_{\mathrm{SI}}\left(u_{1}^{t}=i\right)=p_{\mathrm{sp}} \cdot A_{2}\left(u_{2}^{t}=i\right)+\sum_{j=0, j \neq i}^{M-1} q_{\mathrm{sp}} \cdot A_{2}\left(u_{2}^{t}=j\right)$,

for $i=\{0,1, . ., M-1\}$ and $t=\{1,2, \ldots, N\}$, where $A_{\mathrm{SI}}\left(u_{1}^{t}\right)$ is the a priori symbol probability extracted from the side information. The $M$-SC transition probabilities, $p_{\mathrm{sp}}$ and $q_{\mathrm{sp}}$ are embedded into this correction function $f_{\mathrm{c}}$ with the objective of exploiting the spatial correlation. The transition probabilities can be estimated for example by the algorithm of [53], but in our work we assume that $p_{\mathrm{sp}}$ and $q_{\mathrm{sp}}$ are known to the decoder. The decoding process of $\mathrm{DEC}_{\mathrm{S}}$ seen in Fig. 4 is performed using the MAP algorithm about to be presented in Section III(B), which then provides the extrinsic information $E_{\mathrm{S}}\left(\mathbf{u}_{1}\right)$. The exchange of extrinsic information between $\mathrm{DEC}_{\mathrm{S}}$ and $\left(\mathrm{DEC}_{\mathrm{U}}+\mathrm{DEC}_{\mathrm{T}}\right)$ of Fig. 4 is defined as the outer iteration and this process is repeated for $\mathcal{I}_{\text {out }}$ number of times. At the end of each outer iteration, the a priori information of $A_{\mathrm{T}}(\mathbf{v})$ and $A_{\mathrm{U}}\left(\mathbf{v}^{\prime}\right)$ is reset to zero, before proceeding with the next round of $\mathcal{I}_{\text {in }}$ inner iterations. Following the final outer iteration, a hard-decision is performed based on the a posteriori information output of $\mathrm{DEC}_{\mathrm{S}}$ for obtaining the estimated information symbol sequence $\hat{\mathbf{u}}_{1}$. The proposed scheme can also be invoked for exploiting spatial-only correlation (defined as DJSUTCM-S) and in this case, $\mathrm{DEC}_{\mathrm{S}}$ (as well as $\Pi_{\mathrm{ST}}$ and $\Pi_{\mathrm{ST}}^{-1}$ ) of Fig. 4 are not needed, since the sources are now memoryless sources exhibiting no correlation in the time domain. The side information $A_{\mathrm{SI}}\left(\mathbf{u}_{1}\right)$ can then be directly fed into $\mathrm{DEC}_{\mathrm{T}}$ as $A_{\mathrm{T}}\left(\mathbf{u}_{1}\right)$. 


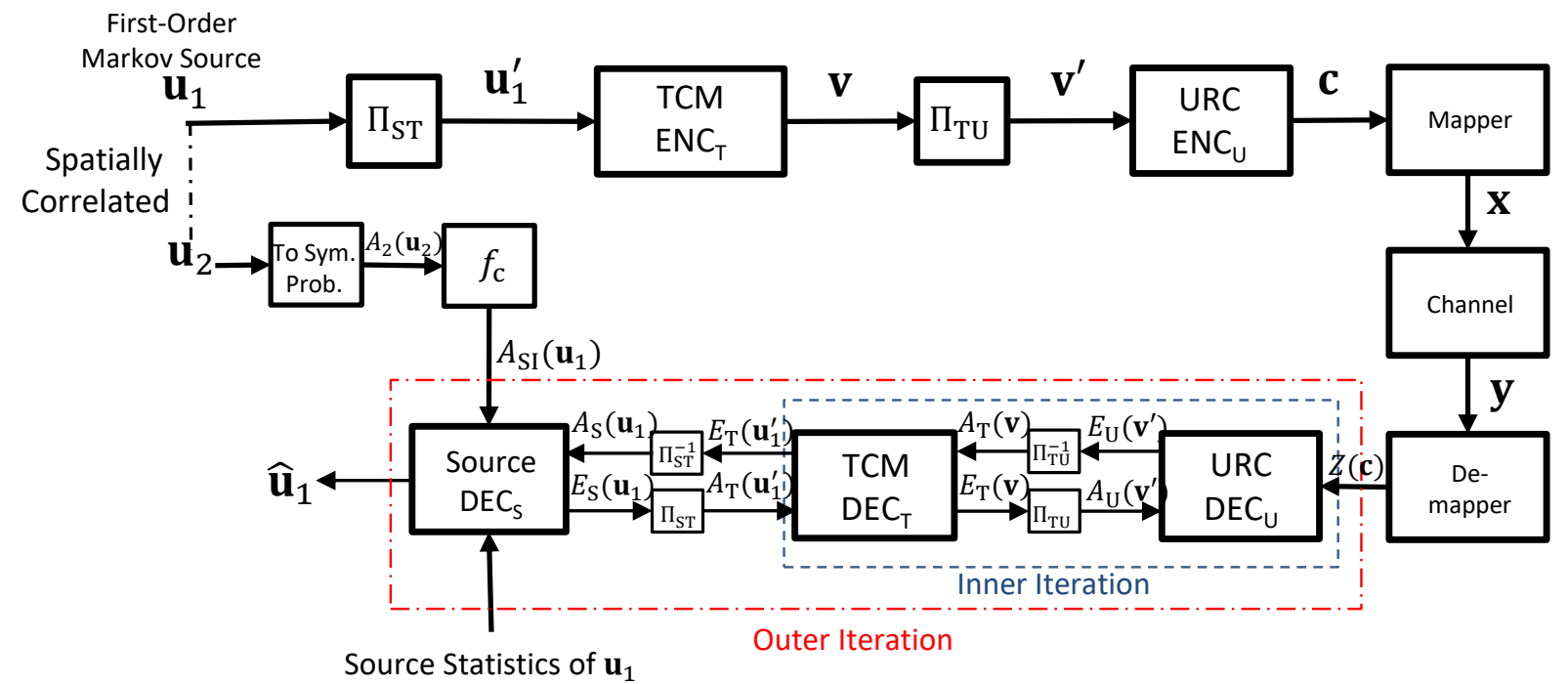

Fig. 4. Block diagram of the proposed symbol-based DJSUTCM-ST scheme exploiting the spatio-temporal correlation.

\section{B. MAP algorithm for First-Order Markov Chain}

The temporal correlation can be readily exploited by the MAP based Markov decoding technique of [33], [54] for soft-bit source decoding. The trellis diagram of the source consists of $M$ trellis states and each state has $M$ legitimate transitions. The transition probabilities of the Markov source are incorporated into the decoding algorithm for exploiting the Markovianity properties of the source. Based on the algorithm of [33], symbol-based source decoding is proposed by replacing the LLR input and output in [33] by the symbol probability input and output, respectively which is more relevant to our symbol-based coding scheme.

At a time index $t$, the Markovianity property inherent in the model yields the a posteriori probability that is associated with the state transition from $u_{1}^{t-1}=i^{\prime}$ to $u_{1}^{t}=i$ with $i^{\prime}, i \in$ $\{0,1, \ldots, M-1\}$ is given by [33]

$$
P\left(u_{1}^{t}=i \mid y^{t}\right)=\alpha_{t}(i) \cdot \gamma_{t}(i) \cdot \beta_{t}(i),
$$

where $y^{t}$ is the received signal at time index $t$. The forward recursive coefficient $\alpha_{t}$ and the backward recursive coefficient $\beta_{t}$ are computed recursively by [33]

$$
\alpha_{t}(i)=\sum_{i^{\prime}} \alpha_{t-1}\left(i^{\prime}\right) \cdot \gamma_{t-1}\left(i^{\prime}\right) \cdot a_{i^{\prime}, i}^{\mathrm{te}}
$$

and

$$
\beta_{t}\left(i^{\prime}\right)=\sum_{i} \beta_{t+1}(i) \cdot \gamma_{t+1}(i) \cdot a_{i^{\prime}, i}^{\mathrm{te}}
$$

respectively, where $a_{i^{\prime}, i}^{\text {te }}$ is the transition probability given in (4). Meanwhile, $\gamma_{t}(i)$ is the overall a priori symbol probability formulated as

$$
\gamma_{t}(i)=c_{\gamma_{t}} \cdot A_{\mathrm{SI}}\left(u_{1}^{t}=i\right) \cdot A_{\mathrm{S}}\left(u_{1}^{t}=i\right),
$$

where $c_{\gamma_{t}}$ is the normalization factor, which solely depends on $y^{t}$. The extrinsic output $E_{\mathrm{S}}\left(u_{1}^{t}\right)$ can be computed by removing the a priori information $A_{\mathrm{S}}\left(u_{1}^{t}\right)$ from the a posteriori probability $P\left(u_{1}^{t} \mid y^{t}\right)$ in (25).

\section{NuMERICAL RESULTS}

\section{A. EXIT chart analysis}

Symbol-based EXIT charts [55], [56] were used for evaluating the exchange of mutual information between the decoders in the proposed system. In this work, we evaluate the EXIT characteristics of $\mathbf{u}_{1}$ between $\mathrm{DEC}_{\mathrm{S}}$ of Fig. 4 as the outer decoder and the combination of $\mathrm{DEC}_{\mathrm{U}}+\mathrm{DEC}_{\mathrm{T}}$ as the inner decoder. The spatio-temporal correlation is exploited at $\mathrm{DEC}_{\mathrm{S}}$ and it is expected that an extrinsic mutual information improvement can be achieved by exploiting the spatio-temporal correlation. The EXIT function $T_{\text {out }}($.$) of \mathrm{DEC}_{S}$ and of $T_{\text {in }}($. for $\mathrm{DEC}_{\mathrm{U}}+\mathrm{DEC}_{\mathrm{T}}$ are given as

$$
I_{\mathrm{E}, \text { out }}=T_{\mathrm{out}}\left(I_{\mathrm{A}, \text { out }}, \rho_{\mathrm{sp}}, \rho_{\mathrm{te}}\right)
$$

and

$$
I_{\mathrm{E}, \text { in }}=T_{\text {in }}\left(I_{\mathrm{A}, \text { in }}, E_{\mathrm{so}} / N_{0}, \mathcal{I}_{\text {in }}\right),
$$

respectively, where $I_{\mathrm{E} \text {,out }}$ and $I_{\mathrm{A} \text {,out }}$ are the extrinsic and the a priori mutual information of $\mathbf{u}_{1}$ for $\mathrm{DEC}_{\mathrm{S}}$, respectively. Meanwhile, $I_{\mathrm{E}, \text { in }}$ and $I_{\mathrm{A} \text {,in }}$ are the extrinsic and a priori mutual information for $\mathrm{DEC}_{\mathrm{U}}+\mathrm{DEC}_{\mathrm{T}}$, respectively. It can be observed in (30) that the value of $I_{\mathrm{E} \text {,in }}$ is affected by $\mathcal{I}_{\text {in }}$ since we can expect better $I_{\mathrm{E} \text {,in }}$ resulted for an increased number of inner iterations. In our overall simulations, we fixed $\mathcal{I}_{\text {in }}=15$ since we observed no significant improvement beyond that.

The EXIT characteristics of $\mathrm{DEC}_{\mathrm{S}}$ are depicted in Fig. 5 for sources having $R_{\mathrm{s}}=2$. In the case of $\rho_{\mathrm{te}}=0$ when only the spatial correlation is exploited, a constant $I_{\mathrm{E} \text {,out }}$ value is observed for all $I_{\mathrm{A} \text {,out }}$ values. This is not unexpected since the source decoder is designed for exploiting the time domain correlation of first-order Markov sources. However, increasing the spatial correlation strength (corresponding to the value of $\rho_{\mathrm{sp}}$ ) from 0.4 to 0.8 at $I_{\mathrm{A} \text {,out }}=0$ would increase the constant $I_{\mathrm{E}, \text { out }}$ value from 0.29 bit to 1.15 bits. Meanwhile, exploiting the spatio-temporal correlation further improves $I_{\mathrm{E} \text {,out }}$. At $I_{\mathrm{A} \text {,out }}=0, I_{\mathrm{E} \text {,out }}=1.29$ bits improved to 1.69 bits 


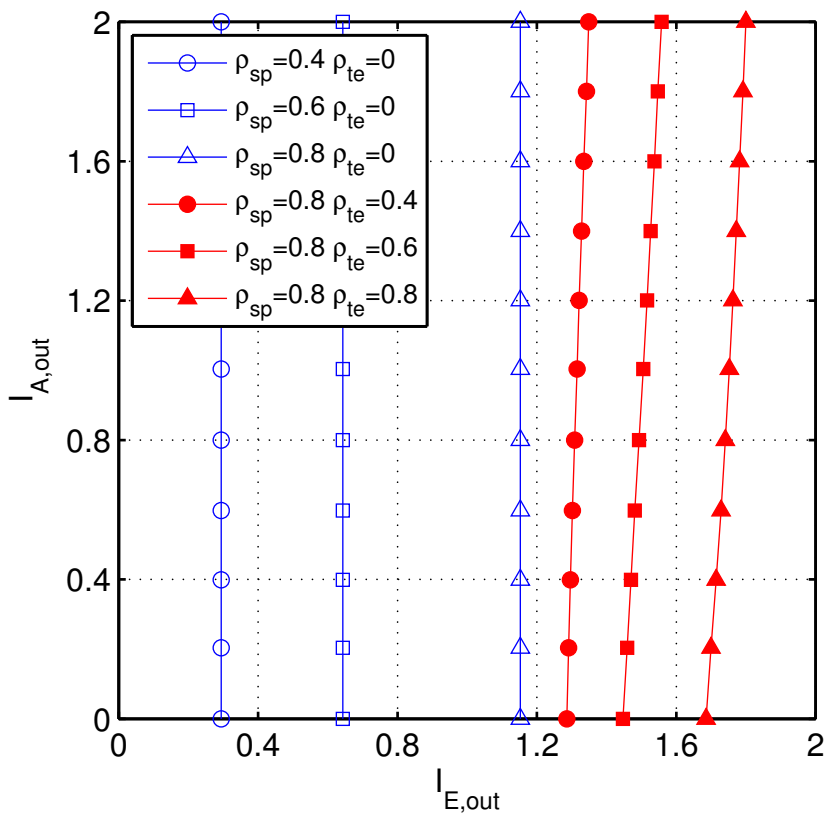

Fig. 5. EXIT characteristics of $\mathrm{DEC}_{\mathrm{S}}$ for sources having spatial-only correlation $\rho_{\mathrm{sp}}=\{0.4,0.6,0.8\}$ (corresponding to $p_{\mathrm{sp}}=\{0.55,0.70,0.85\}$ ) and for sources with spatio-temporal correlation of $\rho_{\mathrm{sp}}=0.8$ and $\rho_{\mathrm{te}}=$ $\{0.4,0.6,0.8\}$ (corresponding to $p_{\mathrm{te}}=\{0.55,0.70,0.85\}$ ).

from sources having $\rho_{\mathrm{te}}=0.4$ to sources having $\rho_{\mathrm{te}}=0.8$ and both sources having $\rho_{\mathrm{sp}}=0.8$.

Fig. 6 compares the EXIT curves of the URC+TCM decoder and TTCM decoder ${ }^{5}$ for various code memory lengths as well as $R_{\mathrm{s}}=2$ and 8PSK modulation. The generator polynomial (GP) of TCM or of the TCM component of TTCM can be expressed as $\left[\begin{array}{lllll}g_{r} & g_{1} & g_{2} & \ldots & g_{R_{\mathrm{s}}+1}\end{array}\right]$, where $g_{r}$ is the feedback generator polynomial, whereas, $g_{k}$ for $k=\left\{1,2, \ldots, R_{\mathrm{s}}+1\right\}$ represents the feed-forward generator polynomial corresponding to the first, second, ..., and $R_{\mathrm{s}}+1$-th input bit, respectively. It can be observed from Fig. 6 that the proposed URC+TCM coding scheme exhibits a better EXIT curve matching with the source decoder than TTCM, since the curves of both 4-state and 8-state TTCM intersect with that of the source decoder. On the other hand, convergence to a vanishingly low symbol error rate (SER) can be expected for all curves of URC+TCM, but for 2-state TCM, a high error-floor is exhibited, although it starts to converge at the lowest $E_{\mathrm{so}} / N_{0}$ threshold amongst the schemes. In this case, URC+4-state TCM would be the preferred choice for the inner code since it has a lower convergence threshold in terms of $E_{\mathrm{so}} / N_{0}$ than URC+8-state TCM and an infinitesimally error floor can be expected at this point. Due to the sharp EXIT curve of $\mathrm{DEC}_{S}$, four or five outer iterations are sufficient to reach the maximum $I_{\mathrm{E}, \text { in }}$ value, as indicated by the Monte-Carlo simulation-based decoding trajectory in Fig. 6. Thus, we opted for $\mathcal{I}_{\text {in }}=15$ and $\mathcal{I}_{\text {out }}=5$ in our overall simulation results.

The EXIT chart of Fig. 6 was recorded for a strong correlation of $\rho_{\mathrm{sp}}=\rho_{\mathrm{te}}=0.8$. However, we found that for weak correlation, the URC+8-state TCM scheme constitutes a the better code of choice due to the high error floor of URC+4-

${ }^{5}$ TTCM decoder was employed in [22] to exploit the spatial correlation

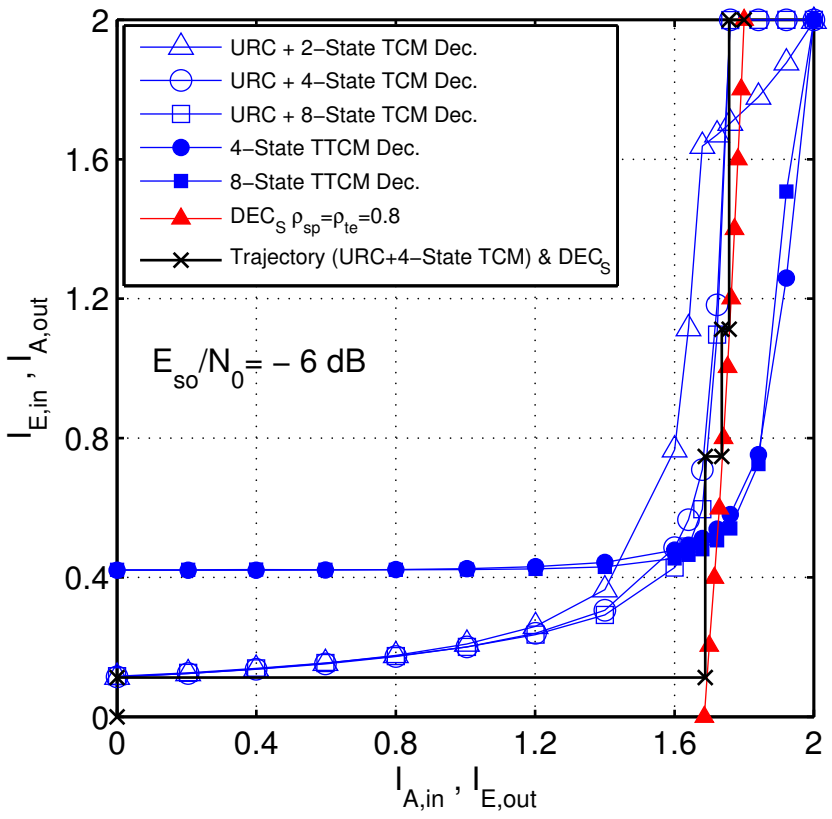

Fig. 6. EXIT chart comparison between the proposed URC+TCM and TTCM for different number of trellis states evaluated at $E_{\mathrm{so}} / N_{0}=-6 \mathrm{~dB}$. Based on the GPs suggested for 8PSK TCM/TTCM in [57], GPs (in octal notation) of $\left[\begin{array}{lll}3 & 2 & 0\end{array}\right]_{8}$ were employed for 2-state TCM, $\left[\begin{array}{lll}7 & 2 & 4\end{array}\right]_{8}$ for 4 -state TCM (and TTCM) and $\left[\begin{array}{lll}13 & 2 & 4\end{array}\right]_{8}$ for 8-state TCM (and TTCM).

TABLE I

THE PROPOSED GPS FOR TCM FOR SOURCES WITH DIFFERENT SYMBOL-LENGTHS

\begin{tabular}{|c|c|c|c|}
\hline \multirow{2}{*}{$R_{\mathrm{s}}$} & \multirow{2}{*}{ Modulation } & \multicolumn{2}{|c|}{ No. of States / GP } \\
\hline & & $\bar{\rho}<\rho_{\mathrm{Th}, R_{\mathrm{S}}}$ & $\bar{\rho} \geq \rho_{\mathrm{Th}, R_{\mathrm{s}}}$ \\
\hline 1 & QPSK & 4-state / $\left[\begin{array}{ll}7 & 4\end{array}\right]_{8}$ & 2-state / [ $\left[\begin{array}{ll}3 & 2\end{array}\right]_{8}$ \\
\hline 2 & 8PSK & 8 -state / $\left[\begin{array}{lll}13 & 2 & 4\end{array}\right]_{8}$ & 4-state / $\left[\begin{array}{lll}7 & 2 & 4\end{array}\right]_{8}$ \\
\hline 3 & 16QAM & 16-state / $\left[\begin{array}{llll}27 & 2 & 4 & 10\end{array}\right]_{8}$ & 8 -state / $\left[\begin{array}{llll}11 & 2 & 4 & 10\end{array}\right]_{8}$ \\
\hline 4 & 32QAM & 16-state / [3 & $\left.2 \begin{array}{lll}4 & 10 & 20\end{array}\right]_{8}$ \\
\hline
\end{tabular}

state TCM. Hence, the correlation coefficient threshold $\rho_{\mathrm{Th}, R_{\mathrm{s}}}$ was determined for $R_{\mathrm{s}}=2$ empirically from EXIT charts in order to determine the condition when to use the URC+4state TCM and URC+8-state TCM. We found that when the average correlation coefficient, $\bar{\rho}=\left(\rho_{\mathrm{sp}}+\rho_{\mathrm{te}}\right) / 2$ is lower than $\rho_{\mathrm{Th}, 2}=0.3$ then URC+8-state $\mathrm{TCM}$ is preferred for the inner code, whereas, if $\bar{\rho} \geq \rho_{\mathrm{Th}, 2}, \mathrm{URC}+4$-state TCM is suggested. In Table I, we provide the suggested GPs for TCM for $R_{\mathrm{S}}=\{1,2,3,4\}$, where it is found that $\rho_{\mathrm{Th}, 1}=0.6$, $\rho_{\mathrm{Th}, 2}=0.3, \rho_{\mathrm{Th}, 3}=0.2$ and $\rho_{\mathrm{Th}, 4}=0$ (since the same GP is proposed regardless of the source correlation for $\left.R_{\mathrm{s}}=4\right)$. The GPs that were included in our search list however are limited to the GPs proposed in [57]-[59] for conventional TCM/TTCM without exploiting the source correlation.

\section{B. SER performance and rate region analysis}

A series of computer simulations was performed for evaluating the proposed DJSUTCM-ST scheme. We conducted simulations using a frame length of $N=10,000$ symbols and 5,000 frames were simulated for each $E_{\text {so }} / N_{0}$ point. The number of trellis states and GPs for TCM used in the simulations were based on the list provided in Table I. Fig. 7 compares 


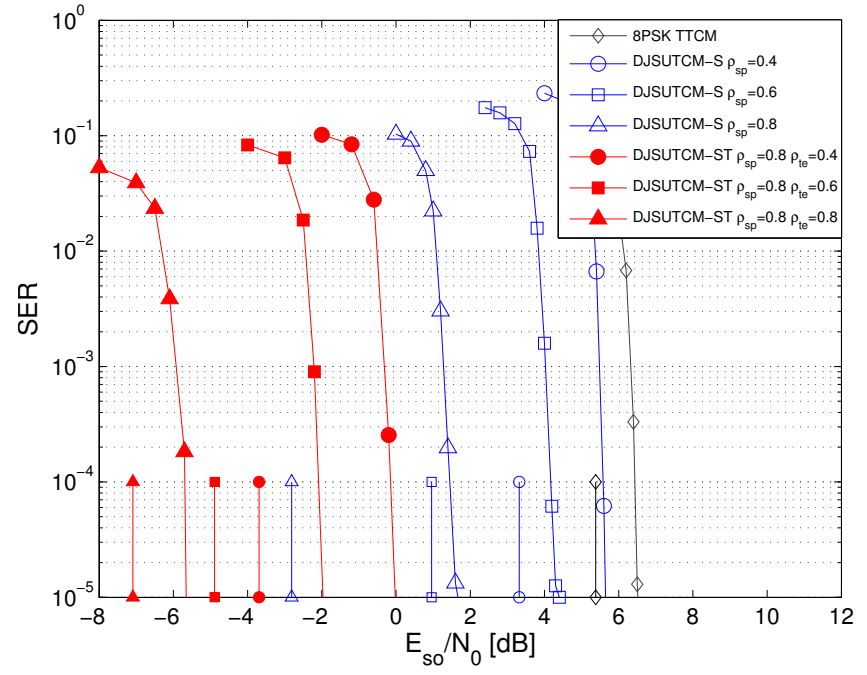

Fig. 7. SER performance of the proposed DJSUTCM-ST exploiting the spatio-temporal correlation, of the DJSUTCM-S exploiting spatial-only correlation and of the conventional 8PSK TTCM for $R_{\mathrm{s}}=2$ bits/symbol sources. The vertical lines indicate the associated capacities

the SER performance of the proposed DJSUTCM-ST scheme exploiting the spatio-temporal correlation with the aid of our DJSUTCM-S scheme that exploits spatial-only correlation and the conventional 8PSK 8-state TTCM (based on the nearcapacity TTCM design of [57]) for sources having $R_{\mathrm{s}}=2$ and having various correlations. Significant performance gains are achieved by using the proposed DJSUTCM-ST scheme over both benchmark schemes, namely over the DJSUTCM$\mathrm{S}$ scheme that relies on spatial-only correlation and over the conventional 8PSK TTCM. In both the DJSUTCM-ST and DJSUTCM-S schemes, a better performance is attained upon relying on stronger source correlation. For example, as can be observed in DJSUTCM-ST, from $E_{\mathrm{so}} / N_{0}=-0.08 \mathrm{~dB}$ at an SER of $10^{-4}$ for sources having $\rho_{\mathrm{sp}}=0.8$ and $\rho_{\mathrm{te}}=0.4$ to $E_{\mathrm{so}} / N_{0}=-5.65 \mathrm{~dB}$ at an SER of $10^{-4}$ for sources associated with $\rho_{\mathrm{sp}}=0.8$ and $\rho_{\mathrm{te}}=0.8$.

The performance gain at an SER of $10^{-4}$ over the conventional 8PSK TTCM and the distance from the theoretical limit extracted from the results of Fig. 7 are summarized in Table II. For conventional 8PSK TTCM, memoryless and uniform sources are assumed and therefore $\mathcal{H}\left(\mathbf{u}_{1} \mid \mathbf{u}_{2}\right)=$ $\mathcal{H}\left(\mathbf{u}_{1}\right)=H\left(u_{2}^{t} \mid u_{1}^{t-1}\right)=2$ bits resulting in $\eta=2$. For spatio-temporally correlated sources, the entropy is reduced as the correlation becomes stronger and correspondingly, a lower $\left(E_{\mathrm{so}} / N_{0}\right)_{\mathrm{lim}}$ value is resulted. It can be observed that for DJSUTCM-S, the distance from the SW/S limit becomes further for sources associated with stronger correlation from $2.28 \mathrm{~dB}$ for sources with $\rho_{\mathrm{sp}}=0.4$ to $4.32 \mathrm{~dB}$ for sources with $\rho_{\mathrm{sp}}=0.8$. However, when exploiting the spatio-temporal correlation using our DJSUTCM-ST scheme, the performance approaches the SW/S limit for stronger source correlation. This is exemplified by DJSUTCM-ST with $\rho_{\mathrm{sp}}=0.8$ and $\rho_{\mathrm{te}}=0.4$ which has an SER curve $3.61 \mathrm{~dB}$ away from the SW/S limit, while DJSUTCM-ST associated with $\rho_{\mathrm{sp}}=0.8$ and $\rho_{\mathrm{te}}=0.8$ has a performance curve $1.44 \mathrm{~dB}$ away from the limit.

The performance of DJSUTCM-S and DJSUTCM-ST for

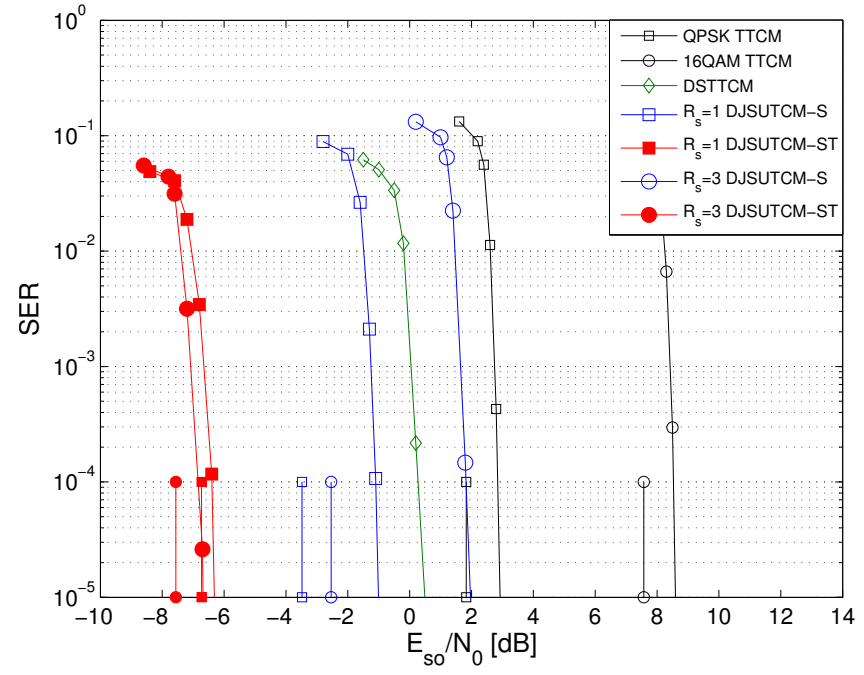

Fig. 8. SER performance of DJSUTCM-S and DJSUTCM-ST for $R_{\mathrm{S}}=1$ and $R_{\mathrm{s}}=3$ bits/symbol sources having $\rho_{\mathrm{sp}}=0.8$ and $\rho_{\mathrm{te}}=0.8$. The vertical lines indicate the associated capacities.

$1 \mathrm{bit} / \mathrm{symbol}$ and $3 \mathrm{bits} / \mathrm{symbol}$ sources having $\rho_{\mathrm{sp}}=0.8$ and $\rho_{\mathrm{te}}=0.8$ is depicted in Fig. 8. The conventional QPSK and 16QAM TTCM were also included as a benchmark for the proposed coding schemes based on $R_{\mathrm{s}}=1$ and $R_{\mathrm{S}}=3$, respectively. An 8-state TTCM with GP $\left[\begin{array}{ll}13 & 6\end{array}\right]_{8}$ was employed for the conventional QPSK TTCM, whereas, a 16-state TTCM with GP [27 24 10 $]_{8}$ was employed for the conventional 16-QAM TTCM [57]. The proposed DJSUTCM$\mathrm{S}$ scheme operating at $R_{\mathrm{s}}=1$ is compared to the TTCM-based coding scheme termed as DSTTCM, which was proposed for exploiting spatial-only correlation for binary sources in [22]. In this case, we set the puncturing rate of the DSTTCM scheme to unity in order to employ the same modulation scheme as our proposed DJSUTCM-S scheme. It can be seen in Fig. 8 for binary sources $\left(R_{s}=1\right)$ that our DJSUTCM$\mathrm{S}$ arrangement outperforms the existing DSTTCM scheme by about $1.2 \mathrm{~dB}$ and has a performance closer to the SW/S limit. This performance advantage is achieved by using only 4-state TCM, while 8-state TTCM was employed by the DSTTCM scheme.

The summary of the performance gains over the conventional TTCM scheme and the distance from the SW/S limit for $R_{\mathrm{S}}=\{1,2,3\}$ bits/symbol sources are tabulated in Table III. Similar to the findings shown in Table II, the DJSUTCM-ST scheme exploiting the spatio-temporal correlation has a performance closer to the theoretical limit than the DJSUTCM-S scheme. In both schemes, sources with $R_{\mathrm{s}}=1$ and QPSK signalling achieve a performance closest to the theoretical limit, outperforming the other sources associated with $R_{\mathrm{s}}=2$ (8PSK signalling) and $R_{\mathrm{s}}=3$ (16QAM signalling). Explicitly, DJSUTCM-S with $R_{\mathrm{s}}=1$ has a performance of $2.40 \mathrm{~dB}$ away from the limit and DJSUTCM-ST with $R_{\mathrm{s}}=1$ performs within $0.35 \mathrm{~dB}$ of the limit. Meanwhile, better $E_{\mathrm{so}} / N_{0}$ gain is achieved for sources having more bits/symbol. More specifically, the DJSUTCM-S scheme outperforms the conventional TTCM by $4.04 \mathrm{~dB}$ and $6.70 \mathrm{~dB}$ for $R_{\mathrm{s}}=1$ and $R_{\mathrm{s}}=3$, 
TABLE II

GAIN IN PERFORMANCE OVER THE CONVENTIONAL 8PSK TTCM AND DISTANCE FROM THE SW/S LIMIT FOR $R_{\mathrm{S}}=2$ BITS/SYMBOL SOURCES

\begin{tabular}{|l|c|c|c|c|c|}
\hline Coding Scheme & $\eta$ & $\left(E_{\mathrm{so}} / N_{0}\right)_{\lim }(\mathrm{dB})$ & $\left(E_{\mathrm{so}} / N_{0}\right)_{10^{-4}}(\mathrm{~dB})$ & Distance $(\mathrm{dB})$ & Gain $(\mathrm{dB})$ \\
\hline 8PSK TTCM & 2.00 & 5.38 & 6.47 & 1.09 & 0 \\
\hline DJSUTCM-S $\rho_{\mathrm{sp}}=0.4$ & 1.71 & 3.32 & 5.60 & 2.28 & 0.87 \\
\hline DJSUTCM-S $\rho_{\mathrm{sp}}=0.6$ & 1.36 & 0.96 & 4.20 & 3.24 & 2.27 \\
\hline DJSUTCM-S $\rho_{\mathrm{sp}}=0.8$ & 0.85 & -2.81 & 1.51 & 4.32 & 4.96 \\
\hline DJSUTCM-ST $\rho_{\mathrm{sp}}=0.8 \rho_{\mathrm{te}}=0.4$ & 0.75 & -3.69 & -0.08 & 3.61 & 6.55 \\
\hline DJSUTCM-ST $\rho_{\mathrm{sp}}=0.8 \rho_{\mathrm{te}}=0.6$ & 0.62 & -4.86 & -1.84 & 3.02 & 8.31 \\
\hline DJSUTCM-ST $\rho_{\mathrm{sp}}=0.8 \rho_{\mathrm{te}}=0.8$ & 0.43 & -7.09 & -5.65 & 1.44 & 12.12 \\
\hline
\end{tabular}

TABLE III

GAIN IN PERFORMANCE OF DJSUTCM-S AND DJSUTCM-ST WITH $R_{\mathrm{S}}=\{1,2,3\}$ OVER THE CONVENTIONAL $2^{R_{\mathrm{S}}+1}$-LEVEL MODULATION TTCM AND DISTANCE FROM THE SW/S LIMIT WHEN EXPLOITING SOURCE CORRELATION $\rho_{\mathrm{SP}}=0.8$ AND $\rho_{\mathrm{TE}}=0.8$

\begin{tabular}{|l|c|c|c|c|c|c|}
\hline Coding Scheme & $R_{\mathrm{S}}$ & $\eta$ & $\left(E_{\mathrm{so}} / N_{0}\right)_{\lim }(\mathrm{dB})$ & $\left(E_{\mathrm{so}} / N_{0}\right)_{10^{-4}}(\mathrm{~dB})$ & Distance $(\mathrm{dB})$ & Gain $(\mathrm{dB})$ \\
\hline \multirow{3}{*}{ DJSUTCM-S } & 1 & 0.47 & -3.48 & -1.09 & 2.40 & 4.04 \\
\cline { 2 - 8 } & 2 & 0.85 & -2.81 & 1.51 & 4.32 & 4.96 \\
\cline { 2 - 7 } & 3 & 1.16 & -2.54 & 1.87 & 4.41 & 6.70 \\
\hline \multirow{3}{*}{ DJSUTCM-ST } & 1 & 0.26 & -6.73 & -6.38 & 0.35 & 9.33 \\
\cline { 2 - 7 } & 2 & 0.43 & -7.09 & -5.65 & 1.44 & 12.12 \\
\cline { 2 - 7 } & 3 & 0.54 & -7.56 & -6.71 & 0.85 & 15.28 \\
\hline
\end{tabular}

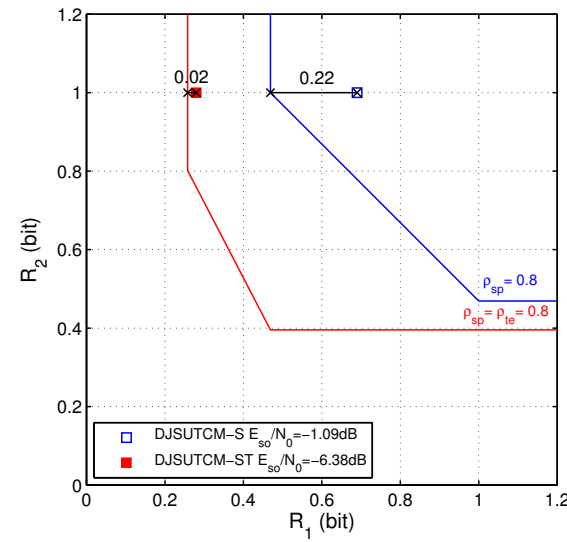

(a) $R_{\mathrm{S}}=1$

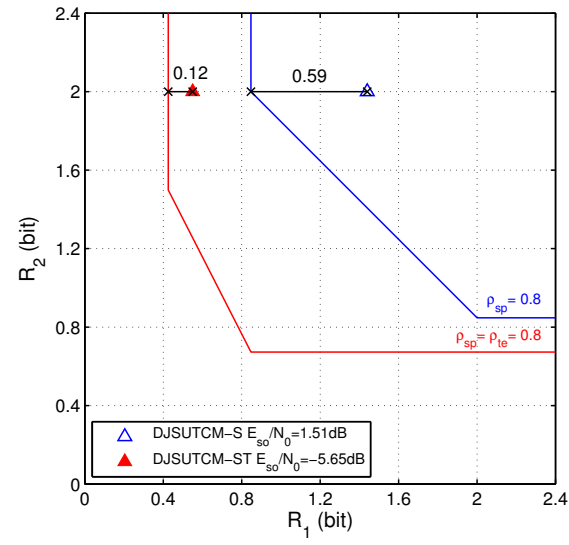

(b) $R_{\mathrm{s}}=2$

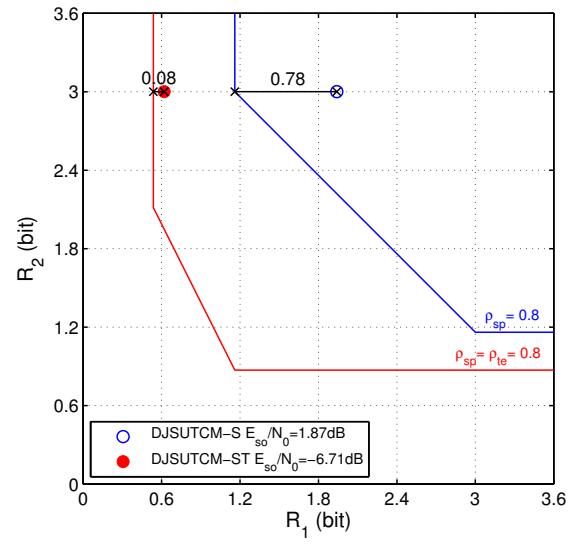

(c) $R_{\mathrm{s}}=3$

Fig. 9. Theoretical SW bound and achievable compression rates obtained using the proposed DJSUTCM-S and DJSUTCM-ST schemes for $R_{\mathrm{S}}=\{1,2,3\}$.

respectively. Similarly, the DJSUTCM-ST scheme outperforms the conventional TTCM by $9.33 \mathrm{~dB}$ and $15.28 \mathrm{~dB}$ for $R_{\mathrm{s}}=1$ and $R_{\mathrm{s}}=3$, respectively.

From the results presented in Table III, the SW bound and the achievable compression rates obtained using the proposed DJSUTCM-S and DJSUTCM-ST schemes are illustrated in Fig. 9 for $R_{\mathrm{s}}=\{1,2,3\}$. As expected, a larger rate region is resulted by exploiting the spatio-temporal correlation compared to spatial-only correlation. In our simulations, the source sequence from Source 2 has a temporal correlation of $\rho_{\mathrm{te} 2}=0.51$ for $R_{\mathrm{s}}=1,2$ and $3 .^{6}$ In this case, since $\mathbf{u}_{2}$ is uncompressed and it is perfectly known at the receiver, we have $R_{2}=R_{\mathrm{S}}$. Hence, the SW-gap with respect to the $\mathrm{SW}$ bound is shown in Fig. 9. It can be observed in Fig. 9 that the proposed DJSUTCM has the smallest gap with respect to the SW bound. Explicitly when $R_{\mathrm{s}}=1$, we have a SW-gap of 0.22 bit and of 0.02 bit, when exploiting the spatial-only and spatio-temporal correlation, respectively.

\footnotetext{
${ }^{6}$ The entropy rate $\mathcal{H}\left(\mathbf{u}_{2}\right)$ corresponding to $R_{\mathrm{S}}=\{1,2,3\}$ are $\{0.80,1.53,2.18\}$ bits.
}

\section{CONCLusions}

In this paper, a symbol-based DJSUTCM scheme exploiting the spatio-temporal correlation has been proposed. A firstorder Markov chain was considered to model the temporal source correlation, while an $M$-SC model was assumed for characterizing the spatial correlation between Source 1 and Source 2. The SW bound was derived for symbol-based sources exhibiting spatio-temporal correlation. In order to exploit the temporal correlation statistics, an iterative decoding process exchanging extrinsic information between URCassisted TCM and a soft-symbol source decoder employing the symbol-based MAP algorithm was invoked. The spatial correlation statistics were exploited by feeding the a priori information from the perfect side information of Source 2 to the receiver of Source 1. Our EXIT chart analysis demonstrated mutual information gains upon exploiting the spatiotemporal correlation using the proposed scheme. We evaluated the SER performance of our DJSUTCM scheme in both cases, relying on spatial-only and spatio-temporal correlation, and demonstrated performance gains over TTCM operating with- 
out exploiting the correlation and the DSTTCM scheme that exploits the spatial-only correlation for binary sources. It was shown that upon exploiting the spatio-temporal correlation of sources having a symbol-length of $1 \mathrm{bit} / \mathrm{symbol}$, the proposed coding scheme is capable of operating within 0.02 bit of the SW bound.

\section{REFERENCES}

[1] D. Slepian and J. Wolf, "Noiseless coding of correlated information sources," IEEE Trans. Inform. Theory, vol. 19, no. 4, pp. 471-480, July 1973.

[2] A. Wyner and J. Ziv, "The rate-distortion function for source coding with side information at the decoder," IEEE Trans. Inform. Theory, vol. 22, no. 1, pp. 1-10, Jan. 1976

[3] S. S. Pradhan and K. Ramchandran, "Distributed source coding using syndromes (DISCUS): design and construction," in Data Compression Conference, 1999. Proceedings. DCC '99, Mar. 1999, pp. 158-167.

[4] — "Distributed source coding using syndromes (DISCUS): design and construction," IEEE Trans. Inform. Theory, vol. 49, no. 3, pp. 626643, Mar. 2003

[5] S. K. Jayaweera, M. L. Chebolu, and R. K. Donapati, "Signalprocessing-aided distributed compression in virtual MIMO-based wireless sensor networks," IEEE Trans. Veh. Technol., vol. 56, no. 5, pp. 2630-2640, Sept 2007.

[6] J. Zheng, P. Wang, and C. Li, "Distributed data aggregation using Slepian-Wolf coding in cluster-based wireless sensor networks," IEEE Trans. Veh. Technol., vol. 59, no. 5, pp. 2564-2574, June 2010.

[7] S. H. Park, O. Simeone, O. Sahin, and S. Shamai, "Robust and efficient distributed compression for cloud radio access networks," IEEE Trans. Veh. Technol., vol. 62, no. 2, pp. 692-703, Feb. 2013.

[8] Y. Qi, M. A. Imran, R. D. Souza, and R. Tafazolli, "On the optimization of distributed compression in multirelay cooperative networks," IEEE Trans. Veh. Technol., vol. 65, no. 4, pp. 2114-2128, Apr. 2016.

[9] J. Bajcsy and P. Mitran, "Coding for the Slepian-Wolf problem with turbo codes," in Global Telecommunications Conference, 2001. GLOBECOM '01. IEEE, vol. 2, 2001, pp. 1400-1404 vol.2.

[10] J. Garcia-Frias, "Compression of correlated binary sources using turbo codes," IEEE Commun. Lett., vol. 5, no. 10, pp. 417-419, Oct. 2001.

[11] A. Aaron and B. Girod, "Compression with side information using turbo codes," in Data Compression Conference, 2002. Proceedings. DCC 2002, 2002, pp. 252-261.

[12] A. D. Liveris, Z. Xiong, and C. N. Georghiades, "A distributed source coding technique for correlated images using turbo-codes," IEEE Commun. Lett., vol. 6, no. 9, pp. 379-381, Sept 2002.

[13] — , "Compression of binary sources with side information at the decoder using LDPC codes," IEEE Commun. Lett., vol. 6, no. 10, pp. 440-442, Oct. 2002.

[14] M. Sartipi and F. Fekri, "Distributed source coding using short to moderate length rate-compatible LDPC codes: the entire Slepian-Wolf rate region," IEEE Trans. Commun., vol. 56, no. 3, pp. 400-411, Mar. 2008.

[15] J. Garcia-Frias, "Joint source-channel decoding of correlated sources over noisy channels," in Proceedings DCC 2001. Data Compression Conference, 2001, pp. 283-292.

[16] J. Garcia-Frias and Y. Zhao, "Near-Shannon/Slepian-Wolf performance for unknown correlated sources over AWGN channels," IEEE Trans. Commun., vol. 53, no. 4, pp. 555-559, Apr. 2005.

[17] J. D. Ser, P. Crespo, and A. Munoz, "Joint source-channel decoding of correlated sources over ISI channels," in Vehicular Technology Conference, 2005. VTC 2005-Spring. 2005 IEEE 61st, vol. 1, May 2005, pp. 625-629 Vol. 1.

[18] Q. Xu, V. Stankovic, and Z. Xiong, "Distributed joint source-channel coding of video using raptor codes," IEEE J. Select. Areas Commun., vol. 25, no. 4, pp. 851-861, May 2007.

[19] K. Anwar and T. Matsumoto, "Iterative spatial demapping for two correlated sources with power control over fading MAC," in Vehicular Technology Conference (VTC Spring), 2012 IEEE 75th, May 2012, pp. $1-7$.

[20] M. Vaezi and F. Labeau, "Distributed source-channel coding based on real-field BCH codes," IEEE Trans. Signal Processing, vol. 62, no. 5, pp. 1171-1184, Mar. 2014.
[21] S. Schwandter, A. G. i Amat, and G. Matz, "Spatially-coupled LDPC codes for decode-and-forward relaying of two correlated sources over the BEC," IEEE Trans. Commun., vol. 62, no. 4, pp. 1324-1337, Apr. 2014.

[22] A. J. Aljohani, S. X. Ng, and L. Hanzo, "TTCM-aided rate-adaptive distributed source coding for Rayleigh fading channels," IEEE Trans. Veh. Technol., vol. 63, no. 3, pp. 1126-1134, Mar. 2014.

[23] — , "Distributed source coding and its applications in relaying-based transmission," IEEE Access, vol. 4, pp. 1940-1970, 2016.

[24] X. Zhou, M. Cheng, K. Anwar, and T. Matsumoto, "Distributed joint source-channel coding for relay systems exploiting sourcerelay correlation and source memory," EURASIP J. Wirel. Commun Netw., vol. 2012, no. 1, pp. 1-13, 2012. [Online]. Available: http://dx.doi.org/10.1186/1687-1499-2012-260

[25] N. Sun, J. Wu, and G. Zhou, "Distributed joint source-channel code for spatial-temporally correlated Markov sources," in Communications (ICC), 2013 IEEE International Conference on, June 2013, pp. 49054910.

[26] R. Asvadi, T. Matsumoto, and M. Juntti, "Joint distributed sourcechannel decoding for LDPC-coded binary Markov sources," in Personal Indoor and Mobile Radio Communications (PIMRC), 2013 IEEE 24th International Symposium on, Sept 2013, pp. 807-811.

[27] A. Zribi and R. Pyndiah, "Cooperative communication using turbo product codes with mutiple-source spatial and temporal correlations,' in Turbo Codes and Iterative Information Processing (ISTC), 2014 8th International Symposium on, Aug. 2014, pp. 249-253.

[28] M. Adrat and P. Vary, "Iterative source-channel decoding: Improved system design using EXIT charts," EURASIP J. Appl. Signal Process., vol. 2005, no. 6, pp. 928-941, May 2005 .

[29] J. Kliewer, N. Görtz, and A. Mertins, "Iterative source-channel decoding with Markov random field source models," IEEE Trans. Signal Processing, vol. 54, no. 10 , pp. $3688-3701$, Oct. 2006.

[30] F. Lahouti, A. K. Khandani, and A. Saleh, "Robust transmission of multistage vector quantized sources over noisy communication channels - applications to MELP speech codec," IEEE Trans. Veh. Technol., vol. 55, no. 6, pp. 1805-1811, Nov. 2006.

[31] Nasruminallah and L. Hanzo, "EXIT-chart optimized short block codes for iterative joint source and channel decoding in H.264 video telephony," IEEE Trans. Veh. Technol., vol. 58, no. 8, pp. 4306-4315, Oct. 2009.

[32] S. Ahmed, R. G. Maunder, L. L. Yang, and L. Hanzo, "Iterative detection of unity-rate precoded FFH-MFSK and irregular variable-length coding," IEEE Trans. Veh. Technol., vol. 58, no. 7, pp. 3765-3770, Sept 2009.

[33] Y. Huo, C. Zhu, and L. Hanzo, "Spatio-temporal iterative source-channel decoding aided video transmission," IEEE Trans. Veh. Technol., vol. 62 , no. 4, pp. 1597-1609, May 2013.

[34] S. Shahidi, F. Alajaji, and T. Linder, "MAP detection and robust lossy coding over soft-decision correlated fading channels," IEEE Trans. Veh. Technol., vol. 62, no. 7, pp. 3175-3187, Sept 2013.

[35] J. Hagenauer, "Source-controlled channel decoding," IEEE Trans. Commun., vol. 43, no. 9, pp. 2449-2457, Sept. 1995

[36] J. Garcia-Frias and J. D. Villasenor, "Combining hidden Markov source models and parallel concatenated codes," IEEE Commun. Lett., vol. 1, no. 4, pp. 111-113, July 1997.

[37] G. Zhu and F. Alajaji, "Joint source-channel turbo coding for binary Markov sources," IEEE Trans. Wireless Commun., vol. 5, no. 5, pp. 1065-1075, May 2006.

[38] M. A. M. Izhar, N. Fisal, X. Zhou, K. Anwar, and T. Matsumoto, "Exploitation of 2D binary source correlation using turbo block codes with fine-tuning," EURASIP J. Wirel. Commun. Netw., vol. 2013, no. 89, pp. 1-11, Mar. 2013.

[39] M. A. M. Izhar, X. Zhou, and T. Matsumoto, "Utilization of multi-dimensional source correlation in multi-dimensional single parity check codes," Telecommunication Systems, vol. 62, no. 4, pp. 735-745, 2016. [Online]. Available: http://dx.doi.org/10.1007/s11235-015-0107-5

[40] L. Bahl, J. Cocke, F. Jelinek, and J. Raviv, "Optimal decoding of linear codes for minimizing symbol error rates (corresp.)," IEEE Trans. Inform. Theory, vol. 20, no. 2, pp. 284-287, Mar. 1974.

[41] J. Proakis and M. Salehi, Digital Communications, 5th ed. McGrawHill Science/Engineering/Math, Nov. 2007.

[42] A. Abrardo, M. Barni, E. Magli, and F. Nencini, "Error-resilient and lowcomplexity onboard lossless compression of hyperspectral images by means of distributed source coding," IEEE Transactions on Geoscience and Remote Sensing, vol. 48, no. 4, pp. 1892-1904, Apr. 2010.

[43] S. Wang, L. Cui, S. Cheng, L. Stankovic, and V. Stankovic, "Onboard low-complexity compression of solar stereo images," IEEE Transactions on Image Processing, vol. 21, no. 6, pp. 3114-3118, June 2012. 
[44] U. Lee, E. Magistretti, M. Gerla, P. Bellavista, and A. Corradi, "Dissemination and harvesting of urban data using vehicular sensing platforms," IEEE Trans. Veh. Technol., vol. 58, no. 2, pp. 882-901, Feb. 2009.

[45] R. Du, C. Chen, B. Yang, N. Lu, X. Guan, and X. Shen, "Effective urban traffic monitoring by vehicular sensor networks," IEEE Trans. Veh. Technol., vol. 64, no. 1, pp. 273-286, Jan. 2015.

[46] H. T. Cheng, H. Shan, and W. Zhuang, "Infotainment and road safety service support in vehicular networking: From a communication perspective," Mechanical Systems and Signal Processing, vol. 25, no. 6, pp. 2020 - 2038, 2011, interdisciplinary Aspects of Vehicle Dynamics. [Online]. Available: http://www.sciencedirect.com/science/article/pii/S0888327010004127

[47] E. Belyaev, A. Vinel, A. Surak, M. Gabbouj, M. Jonsson, and K. Egiazarian, "Robust vehicle-to-infrastructure video transmission for road surveillance applications," IEEE Trans. Veh. Technol., vol. 64, no. 7, pp. 2991-3003, July 2015.

[48] H. Xie, A. Boukerche, and A. A. F. Loureiro, "MERVS: A novel multichannel error recovery video streaming protocol for vehicle ad hoc networks," IEEE Trans. Veh. Technol., vol. 65, no. 2, pp. 923-935, Feb. 2016.

[49] L. Sun, H. Shan, A. Huang, L. Cai, and H. He, "Channel allocation for adaptive video streaming in vehicular networks," IEEE Trans. Veh. Technol., vol. 66, no. 1, pp. 734-747, Jan. 2017.

[50] P. H. Yuan, K. F. Yang, and W. H. Tsai, "Real-time security monitoring around a video surveillance vehicle with a pair of two-camera omniimaging devices," IEEE Trans. Veh. Technol., vol. 60, no. 8, pp. 36033614 , Oct. 2011.

[51] T. M. Cover and J. A. Thomas, Elements of Information Theory 2nd Edition. USA: John Wiley and Sons, 2006.

[52] S. X. Ng, J. Wang, M. Tao, L. L. Yang, and L. Hanzo, "Iteratively decoded variable length space-time coded modulation: Code construction and convergence analysis," IEEE Trans. Wireless Commun., vol. 6, no. 5, pp. 1953-1963, May 2007.

[53] Y. Zhao and J. Garcia-Frias, "Joint estimation and compression of correlated nonbinary sources using punctured turbo codes," IEEE Trans. Commun., vol. 53, no. 3, pp. 385-390, Mar. 2005.

[54] M. Adrat and P. Vary, "Iterative source-channel decoding: Improved system design using EXIT charts," EURASIP J. Adv. Signal Process., vol. 2005, no. 6, pp. 1-14, 2005. [Online]. Available: http://dx.doi.org/10.1155/ASP.2005.928

[55] S. ten Brink, "Convergence behavior of iteratively decoded parallel concatenated codes," IEEE Trans. Commun., vol. 49, no. 10, pp. 17271737, Oct. 2001.

[56] J. Kliewer, S. X. Ng, and L. Hanzo, "Efficient computation of EXIT functions for nonbinary iterative decoding," IEEE Trans. Commun., vol. 54, no. 12, pp. 2133-2136, Dec. 2006.

[57] S. X. Ng, O. R. Alamri, Y. Li, J. Kliewer, and L. Hanzo, "Near-capacity turbo trellis coded modulation design based on EXIT charts and union bounds," IEEE Trans. Commun., vol. 56, no. 12, pp. 2030-2039, Dec. 2008.

[58] G. Ungerboeck, "Channel coding with multilevel/phase signals," IEEE Trans. Inform. Theory, vol. 28, no. 1, pp. 55-67, Jan. 1982.

[59] P. Robertson and T. Worz, "Bandwidth-efficient turbo trellis-coded modulation using punctured component codes," IEEE J. Select. Areas Commun., vol. 16, no. 2, pp. 206-218, Feb. 1998. 


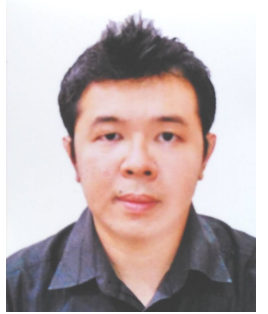

Mohd Azri Mohd Izhar received his M.Eng. degree (First class) in electrical engineering (communications) from the University of Sheffield, U.K. in 2008 and Ph.D. degree in electrical engineering from the Universiti Teknologi Malaysia (UTM), Malaysia in 2014. Since 2014, He has been a senior lecturer with the UTM Kuala Lumpur campus. On leave from the UTM, he was visiting the Southampton Wireless Group, University of Southampton, U.K. for 2 years in 2015 .

His current research interests include channel coding, coding theory, joint source-channel coding, cooperative communications, cognitive radio and quantum error correction codes.

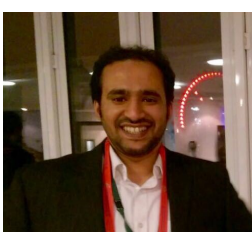

Abdulah Jeza Aljohani received the B.Sc (Eng) degree in electronics and communication engineering from King Abdulaziz University (KAU), Jeddah, Saudi Arabia, in 2006, as well as M.Sc. degree with distinction and Ph.D. degree, awarded with no corrections, in wireless communication from the University of Southampton, Southampton, U.K., in 2010 and 2016, respectively. He is currently working as assistance professor in Electrical Engineering department of Umm Alqura University, Makkah, Saudi Arabia.

His research interests include joint source/channel coding, distributed source coding, coded modulation, channel coding, cooperative communications, as well as MIMO systems.

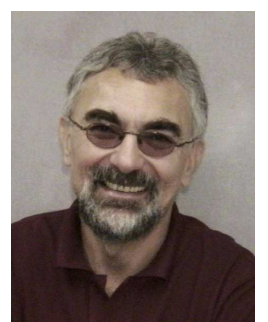

Lajos Hanzo (http://www-mobile.ecs.soton.ac.uk) FREng, FIEEE, FIET, Fellow of EURASIP, DSc received his 5-year Master degree in electronics in 1976, and the Doctoral degree in 1983. In 2009 he was awarded an honorary doctorate by the Technical University of Budapest and in 2015 by the University of Edinburgh. In 2016 he was admitted to the Hungarian Academy of Science. During his 40-year career in telecommunications he has held various research and academic posts in Hungary, Germany and the UK. Since 1986 he has been with the School of Electronics and Computer Science, University of Southampton, UK, where he holds the chair in telecommunications. He has successfully supervised 111 $\mathrm{PhD}$ students, co-authored 18 John Wiley/IEEE Press books on mobile radio communications totalling in excess of 10000 pages, published 1701 research contributions at IEEE Xplore, acted both as TPC and General Chair of IEEE conferences, presented keynote lectures and has been awarded a number of distinctions. Currently he is directing a 60 -strong academic research team, working on a range of research projects in the field of wireless multimedia communications sponsored by industry, the Engineering and Physical Sciences Research Council (EPSRC) UK, the European Research Council's Advanced Fellow Grant and the Royal Society's Wolfson Research Merit Award.

$\mathrm{He}$ is an enthusiastic supporter of industrial and academic liaison and he offers a range of industrial courses. He is also a Governor of the IEEE VTS. During 2008 - 2012 he was the Editor-in-Chief of the IEEE Press and a Chaired Professor also at Tsinghua University, Beijing. For further information on research in progress and associated publications please refer to http://wwwmobile.ecs.soton.ac.uk Lajos has $30000+$ citations and an H-index of 70.

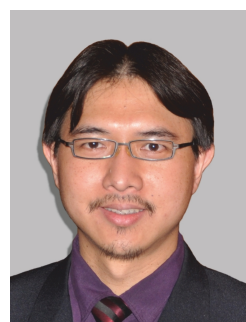

Dr Soon Xin Ng (S'99-M'03-SM'08) received the B.Eng. degree (First class) in electronic engineering and the Ph.D. degree in telecommunications from the University of Southampton, Southampton, U.K., in 1999 and 2002, respectively. From 2003 to 2006, he was a postdoctoral research fellow working on collaborative European research projects known as SCOUT, NEWCOM and PHOENIX. Since August 2006, he has been a member of academic staff in the School of Electronics and Computer Science, University of Southampton. He is involved in the OPTIMIX and CONCERTO European projects as well as the IU-ATC and UC4G projects. He is currently an Associate Professor in telecommunications at the University of Southampton.

His research interests include adaptive coded modulation, coded modulation, channel coding, space-time coding, joint source and channel coding, iterative detection, OFDM, MIMO, cooperative communications, distributed coding, quantum error correction codes and joint wireless-and-optical-fibre communications. He has published over 200 papers and co-authored two John Wiley/IEEE Press books in this field. He is a Senior Member of the IEEE, a Chartered Engineer and a Fellow of the Higher Education Academy in the UK. 